UT-HEP-734-95

\title{
On Quantum Cohomology Rings for Hypersurfaces in $C P^{N-1}$
}

\author{
Masao Jinzenji \\ Department of Physics, University of Tokyo \\ Bunkyo-ku, Tokyo 113, Japan
}

June 11, 2021

\begin{abstract}
Using the torus action method, we construct one variable polynomial representation of quantum cohomology ring for degree $k$ hypersurface in $C P^{N-1}$. The results interpolate the well-known result of $C P^{N-2}$ model and the one of Calabi-Yau hypersuface in $C P^{N-1}$. We find in $k \leq N-2$ case, principal relation of this ring have very simple form compatible with toric compactification of moduli space of holomorphic maps from $C P^{1}$ to $C P^{N-1}$. 由
\end{abstract}

\section{Introduction}

In recent years, study of topological sigma model has made a great progress. In my point of view, these models are classified into following types.

A. topological sigma model (pure matter theory) with the

1. target space $M$ with $c_{1}\left(T^{\prime} M\right)>0$

2. target space $M$ with $c_{1}\left(T^{\prime} M\right)=0$ (Calabi-Yau manifold)

3. target space $M$ with $c_{1}\left(T^{\prime} M\right)<0$

B. topological sigma model coupled to topological gravity with the

1. target space $M$ with $c_{1}\left(T^{\prime} M\right)>0$

2. target space $M$ with $c_{1}\left(T^{\prime} M\right)=0$ (Calabi-Yau manifold)

3. target space $M$ with $c_{1}\left(T^{\prime} M\right)<0$

${ }^{1}$ e-mail address: jin@danjuro.phys.s.u-tokyo.ac.jp 
$T^{\prime} M$ denotes holomorphic part of tangent bundle of Kähler manifold $M$.

For cases A.2 and B.2, we can solve these models using mirror symmetry [9], [10 and [21]. We may also consider the case when the target manifold $M$ is Fano variety, i.e., $c_{1}\left(T^{\prime} M\right) \geq 0$. In [15] and [19], it was shown that models coupled to gravity (small phase space, tree level) can be solved imposing associativity condition of operator algebra. In fact, calculation of amplitudes for general Fano variety by using this method is tedious, but we can't deny the effectiveness of this approach. On the other hand, when target space is $C P^{1}$, the simplest Fano variety, the matrix integral representation of partition function of the model coupled to gravity (large phase space, all genus) was given in [29] by constructing Lax operator formalism of flows induced from insertion of all BRST closed operators. Then we can naturally ask whether we can construct Lax operator representation of sigma models on general Fano varieties. But we don't pursue this problem in this paper.

In this paper we consider the case A.1, topological sigma models on Fano varaeties without coupling to gravity. In this case, Vafa and Intriligator [1] conjectured quantum cohomology rings that correspond to the solutions of sigma models on Grassmanianns. They first considered Landau-Ginzburg potential $W(X)$ that determines the relations (ideal) of classical cohomology ring of Grassmannians. For example, $W(X)$ in $C P^{N}$ model is given by

$$
W^{C P^{N}}(X)=\frac{X^{N+2}}{N+2}
$$

where $X$ represents the Kähler form of $C P^{N}$, the generator of $H^{*}\left(C P^{N}, C\right)$. Then the relation of $H^{*}\left(C P^{N}, C\right)$ is obtained from $\partial_{X} W(X)=0$

$$
\partial_{X} W^{C P^{N}}(X)=X^{N+1}=0 .
$$

From this we can determine ring structure of $H^{*}\left(C P^{N}, C\right)$. Then they argued that relations of the quantum cohomology ring are generated by Landau-Ginsburg potential perturbed along the direction of Kähler form,

$$
W_{q}^{C P^{N}}(X)=\frac{X^{N+2}}{N+2}-X q
$$

where $q\left(:=e^{-t}\right)$ is the deformation parameter which counts the degree of instanton (holomorphic maps from Riemann surface to $C P^{N}$ ). Relation of quantum cohomology ring is derived in the same way as the classical case,

$$
\partial_{X} W_{q}^{C P^{N-1}}(X)=X^{N+1}-q=0 .
$$

For a general Grassmannian $\operatorname{Gr}(N, N+M)$ whose cohomology ring has $N$ generators, the situation is the same. Let $W_{N+M+1}^{(N)}\left(X_{1}, \cdots, X_{N}\right)$ $]$ be the Landau-Ginzburg potential for $\operatorname{Gr}(N, N+M)$, then Landau-Ginzburg potential of quantum cohomology

\footnotetext{
${ }^{2}$ Let $X^{(N)}(t)=\sum_{i=0}^{N} X_{i} t^{i}$. Then $W_{N+M+1}^{(N)}\left(X_{1}, \cdots, X_{N}\right)$ is given by $t^{N+M+1}$ coefficient of $W^{(N)}(t)=-\log \left(X^{(N)}(-t)\right)=\sum_{i} W_{i}^{(N)}\left(X_{1}, \cdots, X_{N}\right) t^{i}$
} 
ring is

$$
W_{q}^{G r(N, N+M)}\left(X_{1}, \cdots, X_{N}\right):=W_{N+M+1}^{(N)}\left(X_{1}, \cdots, X_{N}\right)+(-1)^{N} q X_{1}
$$

and relations of this ring are given by $\partial_{X_{i}} W_{q}^{G r(N, N+m)}=0$. One can evaluate correlation function of these models from the residue formula,

$$
\left\langle\prod_{j=1}^{m} \mathcal{O}_{A_{j}}\left(z_{j}\right)\right\rangle=\oint_{C_{\infty}} \cdots \oint_{C_{\infty}} \frac{\prod_{j=1}^{m} f_{A_{j}}(X)}{\prod_{i=1}^{N}\left(\partial_{X_{i}} W_{q}^{G r(N, N+M)}(X)\right)} d X_{1} \cdots d X_{N}
$$

where $f_{A_{i}}(X)$ represents $A_{i} \in H^{*}(G r(N, N+M), C)$ in Landau-Ginzburg representation of $H^{*}(G r(N, N+M), C)$ and $\mathcal{O}_{A_{i}}$ denotes BRST-closed operator of sigma model induced from $A_{i}$. In this case, $f_{A_{i}}(X)$ receives no quantum correction. Correlation functions evaluated from (6) take integer values and are non-vanishing only if the topological selection rule of sigma model is satisfied.

In this construction, geometrical aspect of sigma models, i.e., counting of instantons from Riemann surface to target space $M$ is not clear. But let us assume the fusion rule of correlation function of pure matter theory which can be derived from taking the position of operator insertion points $z_{1}, z_{2}$ in an infinitesimally small disc, or equivalently from putting $z_{1}, z_{2}$ on one component of a stable curve with one branch point and $z_{3}, \cdots, z_{k}$ on the other.

$$
\left\langle\prod_{j=1}^{m} \mathcal{O}_{A_{j}}\left(z_{j}\right)\right\rangle=\left\langle\mathcal{O}_{A_{1}}\left(z_{1}\right) \mathcal{O}_{A_{2}}\left(z_{2}\right) \mathcal{O}_{A_{a}}\left(z_{s 1}\right)\right\rangle \eta^{a b}\left\langle\mathcal{O}_{A_{b}}\left(z_{s 2}\right) \prod_{j=3}^{m} \mathcal{O}_{A_{j}}\left(z_{j}\right)\right\rangle
$$

In (17), $z_{s 1}$ and $z_{s 2}$ are the positions of the double singularities on each component and $\eta^{a b}$ is the flat metric defined from two point functions of the model which receives no quantum correction.

$$
\begin{aligned}
\eta_{a b}: & =\left\langle\mathcal{O}_{A_{a}}\left(z_{1}\right) \mathcal{O}_{A_{b}}\left(z_{2}\right)\right\rangle=\int_{M} A_{a} \wedge A_{b} \\
\eta^{a b} \eta_{b c} & =\delta_{c}^{a}
\end{aligned}
$$

This relation naturally leads us to consider the algebra defined by

$$
\mathcal{O}_{A_{1}} \cdot \mathcal{O}_{A_{2}}=\left\langle\mathcal{O}_{A_{1}} \mathcal{O}_{A_{2}} \mathcal{O}_{A_{a}}\right\rangle \eta^{a b} \mathcal{O}_{A_{b}}
$$

Note that we can regard this definition as the generalized form of multiplication rule of classical cohomology algebra

$$
A_{1} \cdot A_{2}=\left(\int_{M} A_{1} \wedge A_{2} \wedge A_{a}\right) \eta^{a b} A_{b}
$$

Then the geometrical evaluation of three point functions should reproduce the quantum cohomology algebra of Grassmannians and coincide with the result of LandauGinzburg approach. This line of discussion was done by Bertram and Ruan et al [2], [3]. 
In this paper we treat sigma models on degree $k$ hypersurface in $C P^{N-1}, M_{N}^{k}$. $M_{N}^{k}$ has a unique Kähler form $e$ that descends from the Kähler form of $C P^{N-1}$. We can consider $e$ as the generator of the subring of $H^{*}\left(M_{N}^{k}, C\right)$, which consists of $e^{k}(k=1,2, \cdots, N-2)$. We denote it as $H_{e}^{*}\left(M_{N}^{k}, C\right)$. Classically, $H_{e}^{*}\left(M_{N}^{k}, C\right)$ has the following ring structure.

$$
e^{N-1}=0
$$

One can see that from (2), (11) is the same as the relation of $H^{*}\left(C P^{N-2}, C\right)$. Of course, we can also derive the same ring structure by using classical three point functions and metric,

$$
e^{\alpha} \cdot e^{\beta}=\left(\int_{M_{N}^{k}} e^{\alpha} \wedge e^{\beta} \wedge e^{\gamma}\right) \eta^{\gamma \delta} e^{\delta}
$$

where

$$
\begin{aligned}
\int_{M_{N}^{k}} e^{\alpha} \wedge e^{\beta} \wedge e^{\gamma} & =k \cdot \delta_{\alpha+\beta+\gamma, N-2} \\
\eta^{\alpha \beta} & =\frac{1}{k} \delta_{\alpha+\beta, N-2} .
\end{aligned}
$$

Then our assertion is that quantum version of $H_{e}^{*}\left(M_{N}^{k}, C\right)$ (we denote it as $H_{q, e}^{*}\left(M_{N}^{k}, C\right)$ ) is given by

$$
\mathcal{O}_{e^{\alpha}} \cdot \mathcal{O}_{e^{\beta}}=\left\langle\mathcal{O}_{e^{\alpha}} \mathcal{O}_{e^{\beta}} \mathcal{O}_{e^{\gamma}}\right\rangle \eta^{\gamma \delta} \mathcal{O}_{e^{\delta}}
$$

Degree counting parameter $q$ reveals itself in $\left\langle\mathcal{O}_{e^{\alpha}} \mathcal{O}_{e^{\beta}} \mathcal{O}_{e^{\gamma}}\right\rangle$. This is equivalent to the assumption of restricted fusion rule,

$$
\left\langle\prod_{j=1}^{m} \mathcal{O}_{e^{\alpha_{j}}}\left(z_{j}\right)\right\rangle=\left\langle\mathcal{O}_{e^{\alpha_{1}}}\left(z_{1}\right) \mathcal{O}_{e^{\alpha_{2}}}\left(z_{2}\right) \mathcal{O}_{e^{a}}\left(z_{s 1}\right)\right\rangle \eta^{a b}\left\langle\mathcal{O}_{e^{b}}\left(z_{s 2}\right) \prod_{j=3}^{m} \mathcal{O}_{e^{\alpha_{j}}}\left(z_{j}\right)\right\rangle .
$$

In (15), we assumed that n-point correlation function with $(n-1)$ insertions of $\mathcal{O}_{e^{\alpha}}$ 's and 1 insertion from $\mathcal{O}_{A}\left(A \notin H_{e}^{*}\left(M_{N}^{k}, C\right)\right)$ vanishes. If $N$ is odd, (i.e., $\operatorname{dim}\left(M_{N}^{k}\right)$ is odd) justification of this assumption is easy. Because there is no other analytic class in $H^{*}\left(M_{N}^{k}, C\right)$ (with equal holomorphic and anti-holomorphic form degree) except for elements of $H_{e}^{*}\left(M_{N}^{k}, C\right)$. Then the above correlation function vanishes from topological selection rule. In case $\mathrm{N}$ is even, analytic classes which are not included in $H_{e}^{*}\left(M_{N}^{k}, C\right)$ do appear in $H^{\frac{N}{2}-1, \frac{N}{2}-1}\left(M_{N}^{k}, C\right)$, but numerical results of this paper makes no contradiction with (15).

Then what remains to consider is the evaluation of three point functions. These are computed from two facts :

1. Three point functions of pure matter theory and of theory coupled to gravity coincide.

2. Three point functions of theory coupled to gravity are evaluated by using the torus action method [1]. 
In this way, we determined the ring structure of $H_{q, e}^{*}\left(M_{N}^{k}, C\right)$ and find the quantum generalization of the relation (11) if $k$ is no more than $N-2$.

$$
\mathcal{O}_{e}^{N-1}-k^{k} \mathcal{O}_{e}^{k-1} q=0
$$

By setting $\mathcal{O}_{e}:=X$, we can regard (16) as generalization of (4),

$$
\partial_{X} W_{q}^{M_{N}^{k}}=X^{N-1}-k^{k} X^{k-1} q:=f_{\text {rel }}(X)
$$

which reduces to the result of $C P^{N-2}$ model in $k=1$ case. (16) tells us that for $k \geq 2$ case, the direction of deformation is no longer the one of Kähler operator, and $\mathcal{O}_{e^{\alpha}}(\alpha \geq 2)$ indeed receives quantum correction in this case.

$$
\begin{aligned}
\mathcal{O}_{e^{\alpha}}= & X^{\alpha}-\sum_{d=1}^{\left[\frac{\alpha}{N-k}\right]} \gamma_{\alpha, d}^{N, k} X^{\alpha-(N-k) d} q^{d}:=f_{\alpha}(X) \\
& (\alpha=2, \cdots, N-2)
\end{aligned}
$$

We found some curious relations among $\gamma_{\alpha, d}^{N, k}$, s which seems to suggest that higher quantum corrections $(d \geq 2)$ are written in terms of $\gamma_{\alpha, 1}^{N, k}$, s, i.e., degree 1 instanton corrections. This is natural because relation (17) receives corrections only from degree 1 sector.

With these results we obtain the residue formula of correlation functions with full insertion of elements of $H_{q, e}^{*}\left(M_{N}^{k}, C\right)$.

$$
\left\langle\prod_{j=1}^{m} \mathcal{O}_{e^{\alpha}}\left(z_{j}\right)\right\rangle=k \cdot \oint_{\infty} \frac{\prod_{j=1}^{m} f_{\alpha_{j}}(X)}{f_{r e l}(X)} d X
$$

This is the generalization of (6).

The relation (16) has some geometrical meaning. Using (19), we can easily see

$$
\left\langle\prod_{j=1}^{(N-2)+(N-k) d} \mathcal{O}_{e}\left(z_{j}\right)\right\rangle=k^{k d+1} q^{d}
$$

In [21], we found that correlation function $\left\langle\prod_{j=1}^{N-2} \mathcal{O}_{e}\left(z_{j}\right)\right\rangle=\sum_{d=0}^{\infty}\left\langle\prod_{j=1}^{N-2} \mathcal{O}_{e}\left(z_{j}\right)\right\rangle_{d} q^{d}$ of sigma model on Calabi-Yau hyper surface in $C P^{N-1}\left(M_{N}^{N}\right)$ has the following structure

$$
\left\langle\prod_{i=1}^{N-2} \mathcal{O}_{e}\left(z_{i}\right)\right\rangle_{d}=\left(N^{d N+1}-(\text { correction terms })\right),
$$

using mirror symmetry. We asserted there that the top term $\left(N^{d N+1}\right)$ can be evaluated from simple compactification of moduli space $\mathcal{M}_{0, d}^{C P^{N-1}}$ of instantons (holomorphic maps) from $C P^{1}$ to $C P^{N-1}$ (We compactified $\mathcal{M}_{0, d}^{C P^{N-1}}$ into $C P^{(d+1) N-1}$ ) and that correction terms appear as the effect of boundary components added in the process of compactification. This argument is shown to correspond to the result of 
gauged linear sigma model in [28]. (20) tells us that there are no correction terms in $k \leq N-2$ case. This fact can be explained from the above point of view. Boundary components are irrelevant in this case in view of the dimensional counting.

In section 2, we review fundamental facts of topological sigma model (A-model) for pure matter case, introduce the notion of quantum cohomology algebra and discuss its applications to pure A-model.

In section 3, we briefly explain the strategy of the construction of quantum cohomology ring of $M_{N}^{k}$. In section 4, we reformulate this ring as the polynomial algebra of $\mathcal{O}_{e}$ and discuss its characteristic structure when $k \leq N-2$. In section 5 , we try to understand geometrical reason of the bound $k \leq N-2$ and explain why under this bound boundaries of $C P^{d(N+1)-1}-\mathcal{M}_{0, d}^{C P^{N-1}}$ are irrelevant in integration of forms.

All numerical results used in this paper are collected at the end of this paper.

\section{Topological Sigma Model (A-model)}

Topological sigma model is constructed from twisting $N=2$ super symmetric sigma model that describes maps from Riemann surface $\Sigma$ to Kähler manifold $M$ (In this paper we limit genus of Riemann surface to 0 ). Then we have the following Lagrangian.

$L=2 t \int_{\Sigma} d^{2} z\left(\frac{1}{2} g_{i \bar{j}}\left(\partial_{z} \phi^{i} \partial_{\bar{z}} \phi^{\bar{j}}+\partial_{z} \phi^{\bar{j}} \partial_{\bar{z}} \phi^{i}\right)+i \psi_{z}^{\bar{i}} D_{\bar{z}} \chi^{i} g_{i \bar{i}}+i \psi_{\bar{z}}^{i} D_{z} \chi^{\bar{i}} g_{i \bar{i}}-R_{i \bar{i} j \bar{j}} \psi_{\bar{z}}^{i} \psi_{z}^{\bar{i}} \chi^{j} \chi^{\bar{j}}\right)$

where $\phi$ is the map from $C P^{1}$ to $M$, the only bosonic degrees of freedom of this model, and $\chi$ is fermionic ghost fields with ghost number 1 and $\psi$ are anti-ghosts with ghost number -1 . This Lagrangian is invariant under the BRST-transformation,

$$
\begin{aligned}
\delta \phi^{i} & =i \alpha \chi^{i}, \delta \phi^{\bar{i}}=i \alpha \chi^{\bar{i}} \\
\delta \chi_{i} & =0, \delta \chi_{\bar{i}}=0 \\
\delta \psi_{z}^{\bar{i}} & =-\alpha \partial_{z} \phi^{\bar{i}}-i \alpha \chi^{\bar{j}} \Gamma_{\bar{j} \bar{m}}^{\bar{i}} \psi_{z}^{\bar{m}} \\
\delta \psi_{\bar{z}}^{i} & \left.=-\alpha \partial_{\bar{z}} \phi^{i}-i \alpha \chi^{j} \Gamma_{j m}^{i}\right) \psi_{\bar{z}}^{m} .
\end{aligned}
$$

We define the generator of this transformation as $Q$,i.e., $Q$ acts as $\delta V=-\alpha\{Q, V\}$ for any field $V$. This transformation is nilpotent and we only have to consider BRSTclosed operator as observables of this theory.

BRST-closed observables are constructed from closed form on $M$. Let $A=$ $A_{i_{1} i_{2} \cdots i_{k}} d \phi^{i_{1}} \wedge \cdots \wedge d \phi^{i_{k}}$ be an $k$-form on $M$ and we define a corresponding operator $\mathcal{O}_{A}:=A_{i_{1} i_{2} \cdots i_{k}} \chi^{i_{1}} \cdots \chi^{i_{k}}$. Then from BRST-transformation rules, we have

$$
\left\{Q, \mathcal{O}_{A}\right\}=-\mathcal{O}_{d A}
$$

This tells us that we can construct BRST-closed observable $\mathcal{O}_{A}$ from the element $A$ of de Rham cohomology ring $H^{*}(M)$. 
Of course, Lagrangian $L$ satisfies $\{Q, L\}=0$. Moreover we can rewrite $L$ modulo $\psi$ equation of motion in the following form.

$$
L=i t \int_{\Sigma} d^{z}\{Q, V\}+t \int_{\Sigma} \Phi^{*}(e)
$$

where

$$
\begin{aligned}
V & =g_{i \bar{j}}\left(\psi_{z}^{\bar{i}} \partial_{\bar{z}} \phi^{j}+\partial_{z} \phi^{\bar{i}} \psi_{\bar{z}}^{j}\right) \\
\int_{\Sigma} \Phi^{*}(e) & =\int_{\Sigma}\left(\partial_{z} \phi^{i} \partial_{\bar{z}} \phi^{\bar{j}} g_{i \bar{j}}-\partial_{\bar{z}} \phi^{i} \partial_{z} \phi^{\bar{j}} g_{i \bar{j}}\right)
\end{aligned}
$$

$\int_{\Sigma} \Phi^{*}(e)$ is the integral of pull-back of the Kähler form $e$ of $M$ and equals $\Phi_{*}(\Sigma) \cap$ $P D(e)$ where $P D(W)$ denotes Poincare dual of $W \in H^{*}(M)$. We call $\Phi_{*}(\Sigma) \cap P D(e)$ as the degree of maps and divide the phase space into sectors $B_{d}$ with fixed degree $d$. In $B_{d}$, the second term of (25) is fixed to $t d$. Then since the first term of (25) is BRST-exact, path-integral is invariant under the variation of the coupling constant. We can easily check this by taking infinitesimal variation of $t$. And we can take weak coupling limit $t \rightarrow \infty$. In this limit, the saddle point approximation of path integral becomes exact and the saddle point is given by the fixed point of BRSTtransformation,

$$
\begin{aligned}
\chi^{i} & =0, \quad \chi^{\bar{i}}=0 \\
\psi_{z}^{\bar{i}} & =0, \quad \psi_{\bar{z}}^{i}=0 \\
\partial_{z} \phi^{\bar{i}} & =0, \quad \partial_{\bar{z}} \phi^{i}=0
\end{aligned}
$$

Especially, the saddle point of bosonic degrees of freedom is given by holomorphic map from $C P^{1}$ to $M$. This saddle point has moduli and we denote this moduli space as $\mathcal{M}_{0, d}^{M}$ where is $d$ is the degree of holomorphic maps. We can count the dimension of $\mathcal{M}_{0, d}^{M}$ by Riemann-Roch theorem,

$$
\begin{aligned}
\operatorname{dim}\left(\mathcal{M}_{0, d}^{M}\right) & =\operatorname{dim}\left(H^{0}\left(\Sigma, f^{*}\left(T^{\prime} M\right)\right)\right) \\
& =\operatorname{dim}(M)+d \cdot c_{1}\left(T^{\prime} M\right)+\operatorname{dim}\left(H^{1}\left(\Sigma, f^{*}\left(T^{\prime} M\right)\right)\right) \\
a_{d} & :=\operatorname{dim}(M)+d \cdot c_{1}\left(T^{\prime} M\right)
\end{aligned}
$$

where $f$ denotes holomorphic map from $\Sigma$ to $M$ considered as the point of $\mathcal{M}_{0, d}^{M}$. Correspondingly, $\chi$ zero modes $\left(\chi_{0}\right)$, the number of which is equal to $\operatorname{dim}\left(H^{0}\left(\Sigma, f^{*}\left(T^{\prime} M\right)\right)\right)$, and $\psi$ zero modes $\left(\psi_{0}\right)$, the number of which is equal to $\operatorname{dim}\left(H^{1}\left(\Sigma, f^{*}\left(T^{\prime} M\right)\right)\right)$ occur. Then by integrating over non-zero modes, we obtain effective Lagrangian $L_{e f f}$ containing $\psi_{0}$ and $\chi_{0}$.

Since $L_{\text {eff }}$ conserves ghost number, we have to compensate this ghost number anomaly by external operator insertions. To make situations simpler, we treat observables induced from analytic cohomology elements $A \in H^{i, i}(M, C)$. We define ghost number of $\mathcal{O}_{A}$ as $\operatorname{dim}_{C}(A)=i$. Then cancelation condition of ghost number 
anomaly reduces to

$$
\begin{aligned}
\left\langle\prod_{j=1}^{k} \mathcal{O}_{A_{j}}\left(z_{j}\right)\right\rangle_{d} & =\int d \mathcal{M}_{0, d}^{M} d \chi_{0} d \psi_{0} \exp \left(-L_{e f f}\right) \prod_{j=1}^{k} \mathcal{O}_{w_{j}}\left(z_{j}\right) \neq 0 \\
& \Longrightarrow \sum_{j=1}^{k} \operatorname{dim}_{C}\left(A_{k}\right)=\operatorname{dim}\left(H^{0}\left(\Sigma, f^{*}\left(T^{\prime} M\right)\right)\right)-\operatorname{dim}\left(H^{1}\left(\Sigma, f^{*}\left(T^{\prime} M\right)\right)\right) \\
& =\operatorname{dim}(M)+d \cdot c_{1}\left(T^{\prime} M\right)
\end{aligned}
$$

This is the topological selection rule.

And with this selection rule, we can rewrite $\left\langle\prod_{j=1}^{k} \mathcal{O}_{A_{j}}\left(z_{j}\right)\right\rangle$ into the following form.

$$
\begin{aligned}
\left\langle\prod_{j=1}^{k} \mathcal{O}_{A_{j}}\left(z_{j}\right)\right\rangle & =\sum_{d=0}^{\infty} \delta_{\left(\sum_{j=1}^{k} \operatorname{dim}_{C}\left(A_{j}\right), a_{d}\right)} \int d \mathcal{M}_{0, d}^{M} d \chi_{0} d \psi_{0} \exp \left(-L_{e f f}\right) \prod_{j=1}^{k} \mathcal{O}_{A_{j}}\left(z_{j}\right) \cdot q^{d} \\
& :=\sum_{d=0}^{\infty} \delta_{\left(\sum_{j=1}^{k} \operatorname{dim}_{C}\left(A_{j}\right), a_{d}\right)}\left\langle\prod_{j=1}^{k} \mathcal{O}_{A_{j}}\left(z_{j}\right)\right\rangle_{d} \cdot q^{d} \\
q & :=e^{-t}
\end{aligned}
$$

With this set up, we evaluate path-integral. Note that remaining degrees of freedom is moduli space of holomorphic maps from $\Sigma$ to $M$ and $\psi$ and $\chi$ zero modes. First, we consider the generic case when

$\operatorname{dim}\left(H^{1}\left(\Sigma, f^{*}\left(T^{1,0} M\right)\right)\right)=0$. In this case, $L_{e f f}$. equals zero because it conserves ghost number. By taking $A_{j} \in H^{i, i}(M, C)$ as the form which has delta function support on $P D\left(A_{j}\right)$, we pick up holomorphic maps which satisfies the following condition in integration of moduli space.

$$
f\left(z_{j}\right) \in P D\left(A_{j}\right) \quad(j=1, \cdots, k)
$$

These conditions impose $\operatorname{dim}_{C}\left(A_{j}\right)$ dimensional constraint on $\mathcal{M}_{0, d}^{M}$ for each $j$ because we use $\left(\operatorname{dim}_{C}\left(A_{j}\right)-1\right)$ degrees of freedom to make $f\left(C P^{1}\right)$ intersect $P D\left(A_{j}\right)$ and 1 degrees of freedom to make $f\left(z_{j}\right) \in P D\left(A_{j}\right)$. These constraints kill all the moduli degrees of freedom as we can easily see from (31). Remaining fermionic fields are balanced by the measure of zero modes in the Grassmann integrand, and correlation function results in

$$
\left\langle\prod_{j=1}^{k} \mathcal{O}_{A_{j}}\left(z_{j}\right)\right\rangle_{d}=\sharp\left\{f: \Sigma \stackrel{\text { hol. }}{\mapsto} M \mid \operatorname{deg}(f)=d, \quad f\left(z_{j}\right) \in P D\left(A_{j}\right) \quad(j=1, \cdots, k)\right\}
$$

We can rewrite (34) into more compact form using the evaluation map

$$
\begin{aligned}
\phi_{j}: \mathcal{M}_{0, d}^{M} & \mapsto M \\
f \in \mathcal{M}_{0, d}^{M} & \mapsto f\left(z_{j}\right),
\end{aligned}
$$


as follows.

$$
\left\langle\prod_{j=1}^{k} \mathcal{O}_{A_{j}}\left(z_{j}\right)\right\rangle_{d}=\int_{\mathcal{M}_{0, d}^{M}} \bigwedge_{j=1}^{k} \phi_{j}^{*}\left(A_{j}\right)
$$

Next, we consider the non-generic case when $\operatorname{dim}_{C}\left(H^{1}\left(C P^{1}, f^{*}\left(T^{\prime} M\right)\right)\right) \neq 0$. From Kodaira-Serre duality, we have

$$
\left(H^{1}\left(C P^{1}, f^{*}\left(T^{\prime} M\right)\right)\right)^{*}=H^{0}\left(C P^{1}, K_{C P^{1}} \otimes f^{*}\left(T^{\prime} M\right)\right)=H^{0}\left(C P^{1}, \mathcal{O}_{C P^{1}}(-2) \otimes f^{*}\left(T^{\prime} M\right)\right) .
$$

On the other hand, since $c_{1}\left(f^{*}\left(T^{\prime} M\right)\right)=f^{*}\left(c_{1}\left(T^{\prime} M\right)\right)=d \cdot c_{1}\left(T^{\prime} M\right)$ and $f^{*}\left(T^{\prime} M\right)$ is rank $\operatorname{dim}_{C}(M)$ bundle on $C P^{1}, f^{*}\left(T^{\prime} M\right)$ is decomposed into a direct sum of line bundles on $C P^{1}$ as follows.

$$
\begin{gathered}
f^{*}\left(T^{\prime} M\right)=\bigoplus_{j=1}^{\operatorname{dim}_{C}\left(H^{0}\left(C P^{1}, f^{*}\left(T^{\prime} M\right)\right)\right)}=\sum_{a_{j} \geq 0}\left(a_{j}+1\right)
\end{gathered}
$$

From (37) and (38), we obtain

$$
\mathcal{O}(-2) \otimes f^{*}\left(T^{\prime} M\right)=\bigoplus_{j=1}^{\operatorname{dim}_{C}(M)} \mathcal{O}_{C P^{1}}\left(-2-a_{j}\right)
$$

and

$$
\operatorname{dim}\left(H^{1}\left(C P^{1}, f^{*}\left(T^{\prime} M\right)\right)\right)=\sum_{-2-a_{j} \geq 0}\left(-1-a_{j}\right)
$$

Then we assume the following.

Assumption For an irreducible map $f$ (having no multiple cover map component $), \operatorname{dim}\left(H^{1}\left(C P^{1}, f^{*}\left(T^{\prime} M\right)\right)\right)$ equals 0. And $H^{0}\left(C P^{1}, f^{*}\left(T^{\prime} M\right)\right)$ has at least one $\mathcal{O}_{C P^{1}}(2)$ component that corresponds to an automorphism of $f\left(C P^{1}\right)$ and minimal $\mathcal{O}_{C P^{1}}(-m)$ ( $m$ positive integer) insertion.

Under this assumption, we obtain two cases when $f^{*}\left(T^{\prime}(M)\right)$ has $\mathcal{O}_{C P^{1}}(-m)(m$ positive integer) insertion.

$$
\begin{aligned}
\text { case 1 } f^{*}\left(T^{\prime} M\right)= & \mathcal{O}_{C P^{1}}(2) \oplus \mathcal{O}_{C P^{1}}(-1) \oplus \mathcal{O}_{C P^{1}}(-1) \oplus \mathcal{O}_{C P^{1}}^{d_{M}-3} \\
& \left(c_{1}\left(T^{\prime} M\right)=0, d: \text { arbitrary }\right) \\
\text { case 2 } \quad f^{*}\left(T^{\prime} M\right)= & \mathcal{O}_{C P^{1}}(2) \oplus \mathcal{O}_{C P^{1}}(-1) \oplus \mathcal{O}_{C P^{1}}^{d i m_{M}-2} \\
& \left(c_{1}\left(T^{\prime} M\right)=1, d=1\right)
\end{aligned}
$$

The reason why we pick up the above two cases is that reduced map $\tilde{f}(=f \circ \varphi, \varphi$ is degree $n$ holomorphic map from $C P^{1}$ to $C P^{1}$ ) indeed has non zero $H^{1}$. For these cases, $\tilde{f}^{*}\left(T^{\prime} M\right)$ 's are

case $1 \quad \tilde{f}^{*}\left(T^{\prime} M\right)=\mathcal{O}_{C P^{1}}(2 n) \oplus \mathcal{O}_{C P^{1}}(-n) \oplus \mathcal{O}_{C P^{1}}(-n) \oplus \mathcal{O}_{C P^{1}}^{\text {dim }_{M}-3}$

case $2 \tilde{f}^{*}\left(T^{\prime} M\right)=\mathcal{O}_{C P^{1}}(2 n) \oplus \mathcal{O}_{C P^{1}}(-n) \oplus \mathcal{O}_{C P^{1}}^{\operatorname{dim}_{M}-2}$, 
and $\operatorname{dim} H^{1}$ 's are counted as follows.

$$
\begin{array}{ll}
\text { case } 1 & \operatorname{dim}\left(H^{1}\left(C P^{1}, \tilde{f}^{*}\left(T^{\prime} M\right)\right)\right)=2 n-2 \\
\text { case } 2 & \operatorname{dim}\left(H^{1}\left(C P^{1}, \tilde{f}^{*}\left(T^{\prime} M\right)\right)\right)=n-1
\end{array}
$$

In such cases, these $H^{1}$ 's are stable and we can integrate out corresponding $\psi_{0}$ with weight $\exp \left(-L_{e f f}\right)$. In 30] and [31], it was shown that these integration results in the top Chern class of obstruction bundle $\mathcal{F}\left(H^{1}\right.$ bundle ) on $\mathcal{M}_{0, d, n}^{M}$ (we denote $\mathcal{M}_{0, d, n}^{M}$ as components of moduli space of reduced maps of degree $d$ with $n$ multiple cover). Then we obtain following formula.

$$
\left\langle\prod_{j=1}^{m} \mathcal{O}_{A_{j}}\left(z_{j}\right)\right\rangle_{d}=\sum_{n \mid d} \int_{\mathcal{M}_{0, d, n}^{M}} c_{T}(\mathcal{F}) \wedge\left(\bigwedge_{j=1}^{m} \phi_{j}^{*}\left(A_{j}\right)\right)
$$

Of course, the generic case is included in (44), if we consider $\mathcal{F}$ as rank 0 bundle in this case. In case 1 , we can further reduce $\mathcal{F}$ as a bundle on $\mathcal{M}_{0, n}^{C P^{1}}$ and derive well-known multiple cover formula for models on Calabi-Yau manifolds. In case 2, we cannot argue heuristically that $\mathcal{F}$ as a bundle on $\mathcal{M}_{0, n}^{C P^{1}}$ because in this case, $\operatorname{dim}\left(\mathcal{M}_{0, n}^{C P^{1}}\right)-\operatorname{dim}\left(\left(H^{1}\left(C P^{1}, \tilde{f}^{*}\left(T^{\prime} M\right)\right)\right)\right)=n+2$ and we have nontrivial mixture of the top Chern class and evaluation map contribution. We also have to note that in this case, non trivial contribution should come only from $d=n$ part. Anyway further analysis is needed 35].

Lastly, we explain the notion of the quantum cohomology ring on $M$ as the extension of the classical cohomology rings on $M$. Ring structure of classical cohomology ring on $M$ is determined by the classical three point function $C_{i j k}$ and the metric $\eta^{i j}$ defined by

$$
\begin{aligned}
C_{i j k} & :=\int_{M} A_{i} \wedge A_{j} \wedge A_{k}, \quad \eta_{i j}:=\int_{M} A_{i} \wedge A_{j} \\
\eta_{i j} \eta^{j k} & =\delta_{i}^{k} .
\end{aligned}
$$

In this definition multiplication rules are written as follows

$$
A_{i} \cdot A_{j}=C_{i j k} \eta^{k l} A_{l}
$$

Then we define quantum cohomology ring on $M$. In this ring we change $C_{i j k}$ into $C_{i j k}^{q}$ which we set to three point functions of topological sigma model (A-model) having as world sheet as $C P^{1}$. Metric is unchanged under the assumption of flat metric condition. Then multiplication rules are defined as,

$$
\begin{aligned}
\mathcal{O}_{A_{i}} \cdot \mathcal{O}_{A_{j}} & =C_{i j k}^{q} \eta^{k l} \mathcal{O}_{A_{l}} \\
C_{i j k}^{q} & :=\left\langle\mathcal{O}_{A_{i}}\left(z_{1}\right) \mathcal{O}_{A_{j}}\left(z_{2}\right) \mathcal{O}_{A_{k}}\left(z_{k}\right)\right\rangle
\end{aligned}
$$

Since $\mathcal{M}_{0,0}^{M}$ is the moduli space of constant maps from $C P^{1}$ to $M$, it can be identified with $M$ itself. Then if we expand $C_{i j k}^{q}$ by $q$, we can see the coefficients of $q^{0}$ equals 
classical $C_{i j k}$. In this sense, this algebra is natural extension of the classical cohomology ring on $M$. We can see this algebra is commutative by definition. We assume it is also associative algebra. In the theory coupled with gravity, this condition is powerful to determine correlation functions in the small phase space. 115]

Quantum cohomology ring is an effective notion in treating pure matter theory. Since in pure matter theory, $\left|\mathcal{O}_{A_{i}}\right\rangle$ 's span entire Hilbert space, we can insert identity operator $\left|\mathcal{O}_{A_{i}}\right\rangle \eta^{i j}\left\langle\mathcal{O}_{A_{j}}\right|$ into correlation functions. Especially fusion rules follow from this fact.

$$
\left\langle\mathcal{O}_{A_{i}} \mathcal{O}_{A_{j}} *\right\rangle=\left\langle\mathcal{O}_{A_{i}} \mathcal{O}_{A_{j}} \mathcal{O}_{A_{k}}\right\rangle \eta^{k l}\left\langle\mathcal{O}_{A_{l}} *\right\rangle
$$

This relation can be rewritten using (47) as follows.

$$
\left\langle\mathcal{O}_{A_{i}} \mathcal{O}_{A_{j}} *\right\rangle=\left\langle\left(\mathcal{O}_{A_{i}} \cdot \mathcal{O}_{A_{j}}\right) *\right\rangle
$$

Then by taking product of quantum cohomology ring successively, we can reduce the number of inserted operators to two. In this way correlation functions of pure matter theory having world sheet as $C P^{1}$ are reduced to the problem of determination of all the three point functions.

We make one final remark of this section. If this algebra has some relation $\mathcal{R}\left(\mathcal{O}_{A_{i}}\right)=0$, we can easily see from (49),

$$
\left\langle\mathcal{R}\left(\mathcal{O}_{A_{i}}\right) *\right\rangle=0
$$

So we can compute correlation functions more effectively if we find non-trivial relation of the ring.

\section{Strategy for Determination of Quantum Coho- mology Ring of $M_{N}^{k}$}

In this paper, we treat the topological sigma model with a target space which is the degree $k$ hypersurface $(k \leq N)$ in $C P^{N-1}, M_{N}^{k}$.

$$
M_{N}^{k}:=\left\{\left(X_{1}: X_{2}: \cdots: X_{N}\right) \in C P^{N-1} \mid X_{1}^{k}+\cdots+X_{N}^{k}=0\right\}
$$

Since $M_{N}^{k}$ is a hypersurface of $C P^{N-1}$, we can choose a subring $H_{e}^{*}\left(M_{N}^{k}\right)$ generated by Kä̈ler class $e \in H^{1,1}\left(M_{N}^{k}\right)$. Correspondingly, we assume that BRST-closed observables $\mathcal{O}_{e^{\alpha}} \quad(\alpha=0,1, \cdots, N-2)$ form a closed sub-algebra in quantum cohomology ring of $M_{N}^{k}$ (Operator algebra in pure matter theory). Then we investigate this subalgebra $H_{q, e}^{*}\left(M_{N}^{k}\right)$ in the following way. Operator product algebra is constructed by three point functions and metric as we mentioned in Section 2.

$$
\begin{aligned}
& \mathcal{O}_{e^{\alpha}} \cdot \mathcal{O}_{e^{\beta}}=\left\langle\mathcal{O}_{e^{\alpha}} \mathcal{O}_{e^{\beta}} \mathcal{O}_{e^{\gamma}}\right\rangle \eta^{\gamma \delta} \mathcal{O}_{e^{\delta}} \\
& \eta_{\gamma \delta}:=\left\langle\mathcal{O}_{e^{0}} \mathcal{O}_{e^{\gamma}} \mathcal{O}_{e^{\delta}}\right\rangle=\int_{M_{N}^{k}} e^{\gamma} \wedge e^{\delta}=k \delta_{\gamma+\delta, N-2} \\
& \eta_{\alpha \beta} \eta^{\beta \gamma}=\delta_{\alpha}^{\gamma}
\end{aligned}
$$


Correlation functions in pure matter theory satisfy the fusion rule.

$$
\left\langle\mathcal{O}_{e^{\alpha}} \mathcal{O}_{e^{\beta}} *\right\rangle=\left\langle\mathcal{O}_{e^{\alpha}} \mathcal{O}_{e^{\beta}} \mathcal{O}_{e^{\gamma}}\right\rangle \eta^{\gamma \delta}\left\langle\mathcal{O}_{e^{\delta}} *\right\rangle
$$

In (52) and (53) we assumed that insertion of $\mathcal{O}_{w} \notin H_{q, e}^{*}\left(M_{N}^{k}\right)$ into correlation functions consisting of elements of $H_{q, e}^{*}\left(M_{N}^{k}\right)$ gives zero. This assumption is justified in the case of $M_{N}^{N}$ in [22]. Other cases are discussed in section 1. From the above definition we can easily see $\mathcal{O}_{e^{0}}$ acts trivially on $H_{q, e}^{*}\left(M_{N}^{k}\right)$, and we regard $\mathcal{O}_{e^{0}}$ as identity. Three point functions are determined from the geometrical evaluation of correlation functions of topological sigma model in previous section.

$$
\begin{aligned}
& \left\langle\mathcal{O}_{e^{\alpha}}\left(z_{1}\right) \mathcal{O}_{e^{\beta}}\left(z_{2}\right) \mathcal{O}_{e^{\gamma}}\left(z_{3}\right)\right\rangle \\
& \quad=\sum_{d=0}^{\infty} \delta_{\alpha+\beta+\gamma,(N-k) d+N-2} \int_{\mathcal{M}_{0, d}^{M_{N}^{k}}} c_{T}(\mathcal{F}) \wedge \phi_{1}^{*}\left(e^{\alpha}\right) \wedge \phi_{2}^{*}\left(e^{\beta}\right) \wedge \phi_{3}^{*}\left(e^{\gamma}\right) \cdot q^{d} \\
& \quad=\sum_{d=0}^{\infty} \delta_{\alpha+\beta+\gamma,(N-k) d+N-2} \int_{\mathcal{M}_{0, d, 3}^{M_{N}^{k}}} c_{T}\left(\mathcal{F}^{\prime}\right) \wedge \tilde{\phi}_{1}^{*}\left(e^{\alpha}\right) \wedge \tilde{\phi}_{2}^{*}\left(e^{\beta}\right) \wedge \tilde{\phi}_{3}^{*}\left(e^{\gamma}\right) \cdot q^{d}
\end{aligned}
$$

where

$$
\begin{aligned}
\phi_{i}: \mathcal{M}_{0, d}^{M_{N}^{k}} & \mapsto M_{N}^{k} \phi_{i}(f)=f\left(z_{i}\right) \\
\tilde{\phi}_{i}: \mathcal{M}_{0, d, 3}^{M_{N}^{k}} & \mapsto M_{N}^{k} \tilde{\phi}_{i}\left(\left\{f, z_{1}, z_{2}, z_{3}\right\} / S L(2, C)\right)=f\left(z_{i}\right)
\end{aligned}
$$

$\mathcal{M}_{0, d}^{M_{N}^{k}}$ and $\mathcal{M}_{0, d, 3}^{M_{N}^{k}}$ denote moduli spaces of holomorphic maps of degree $d$ from $C P^{1}$ to $M_{N}^{k}$ of pure matter theory and those of the theory coupled to gravity with three punctures (operator insertion points) respectively. $\mathcal{F}$ and $\mathcal{F}^{\prime}$ are obstruction bundle coming from $H^{1}$. We insert $\delta_{\alpha+\beta+\gamma,(N-k) d+N-2}$ to represent the topological selection rules explicitly.

$$
\begin{aligned}
& \left\langle\prod_{j=1}^{m} \mathcal{O}_{e^{\alpha_{j}}}\left(z_{j}\right)\right\rangle_{d} \neq 0 \\
\Longrightarrow & \sum_{j=1}^{m} \alpha_{j}=\operatorname{dim}_{C}\left(M_{N}^{k}\right)+d \cdot c_{1}\left(T^{\prime} M_{N}^{k}\right)=(N-2)+(N-k) d
\end{aligned}
$$

$c_{1}\left(T^{\prime} M_{N}^{k}\right)$ is calculated from $c\left(T^{\prime} M_{N}^{k}\right)=j^{*}\left(c\left(T^{\prime} C P^{N-1}\right)\right) / j^{*}\left(c\left(\mathcal{O}_{C P^{N-1}}(k H)\right)\right)=(1+$ $e)^{N} /(1+k e)$ where $j$ represents natural embedding from $M_{N}^{k}$ to $C P^{N-1}$ and $e$ is Kähler form of $M_{N}^{k}$.

The equality between the first line and the second line of (54) can be explained as follows. $\mathcal{M}_{0, d}^{M_{N}^{k}}$ has an internal $S L(2, C)$ which moves $\left\{f\left(z_{1}\right), f\left(z_{2}\right), f\left(z_{3}\right)\right\}$ without changing the position of $f\left(C P^{1}\right)$ in $M_{N}^{k}$. In $\mathcal{M}_{0, d, 3}^{M_{N}^{k}}$, these degrees of are killed by dividing by $S L(2, C)$ but the degrees of freedom that change the position of $\left\{z_{1}, z_{2}, z_{3}\right\}$ on $C P^{1}$ are added. Since $S L(2, C)$ can be considered as the degrees of freedom which maps $\{0,1, \infty\}$ to any distinct points $\left\{z_{1}, z_{2}, z_{3}\right\}$, this difference cannot be distinguished under the action of the evaluation maps $\phi_{i}, \tilde{\phi}_{i}$. As to equivalence 
of $c_{T}(\mathcal{F})$ and $c_{T}\left(\mathcal{F}^{\prime}\right)$, we have to rely on numerical results. Equivalence in Calabi-Yau case was examined in [1] and [0].

Then we determine $H_{e, q}^{*}\left(M_{N}^{k}\right)$ with the following strategy.

1. Using the equality of (54), we evaluate all the three point functions using the torus action method with the following equation [5].

$$
\begin{aligned}
\int_{\mathcal{M}_{0, d, 3}^{M_{N}}}^{k} c_{T}\left(\mathcal{F}^{\prime}\right) \wedge \tilde{\phi}_{1}^{*}\left(e^{\alpha}\right) \wedge \tilde{\phi}_{2}^{*}\left(e^{\beta}\right) \wedge \tilde{\phi}_{3}^{*}\left(e^{\gamma}\right) \\
\quad=\int_{\mathcal{M}_{0, d, 3(g \text { ravity })}^{C P N-1}} c_{T}\left(\tilde{\pi}_{3}^{*}\left(\mathcal{E}_{k d+1}\right)\right) \wedge \tilde{\varphi}_{1}^{*}\left(c_{1}^{\alpha}(H)\right) \wedge \tilde{\varphi}_{2}^{*}\left(c_{1}^{\beta}(H)\right) \wedge \tilde{\varphi}_{3}^{*}\left(c_{1}^{\gamma}(H)\right) \\
\quad=\partial_{t_{\alpha}} \partial_{t_{\beta}} \partial_{t_{\gamma}} \operatorname{Res}_{z} \operatorname{Res}_{h}\left(\frac { 1 } { z } \operatorname { l o g } \left(\operatorname{det}\left(\left(g_{i j, j^{\prime} i^{\prime}, d}\right)^{-1}\right) \frac{1}{h} \int d \phi_{i j, d}\right.\right. \\
\quad \exp \left(-\frac{1}{2} \sum_{i, j, d} \frac{-d^{(N-2-(N-k) d)}\left(5^{i} z-5^{j} z\right)^{2} \prod_{l=1}^{N} \prod_{a=1}^{d-1}\left(5^{i} a z+5^{j}(d-a) z-5^{l} d z\right)}{\prod_{a=1}^{k d-1}\left(5^{i} a z+5^{j}(k d-a) z\right)} \phi_{i j, d} \phi_{j i, d}\right. \\
\quad+\sum_{i=1}^{N} \frac{5^{i} k z}{\prod_{j \neq i}\left(5^{i} z-5^{j} z\right)} \sum_{l=1}^{\infty} \frac{1}{l !} \sum_{\substack{d_{1}, \cdots, d_{l}, d_{*} \geq 1 \\
j_{1}, \cdots, j_{l}, j_{*} \neq i}}\left(\frac{v_{i j_{1}, d_{1}}}{z}+\cdots+\frac{v_{i j_{l}, d_{l}}}{z}\right)^{l-3} \phi_{i j_{1}, d_{1}} \cdots \phi_{i j_{l}, d_{l}} \\
\left.\left.\quad \exp \left(\left(5^{i} t_{1} z+\cdots+5^{i(N-2)} t_{N-2} z^{N-2}\right)\left(\frac{v_{i j_{1}, d_{1}}}{z}+\cdots+\frac{v_{i j_{l}, d_{l}}}{z}\right)\right)\right)\right)\left.\right|_{t_{*}=0}
\end{aligned}
$$

where

$$
\begin{aligned}
g_{i j, j^{\prime} i^{\prime}, d} & :=\frac{-d^{(N-2-(N-k) d)}\left(5^{i} z-5^{j} z\right)^{2} \prod_{l=1}^{N} \prod_{a=1}^{d-1}\left(5^{i} a z+5^{j}(d-a) z-d 5^{l} z\right)}{\prod_{a=1}^{k d-1}\left(5^{i} a z+5^{j}(k d-a) z\right)} \\
v_{i j, d} & :=\frac{d}{5^{i}-5^{j}} \\
\tilde{\varphi}_{i} & : \mathcal{M}_{0, d, 3}^{C P^{N-1}} \mapsto C P^{N-1}, \quad \tilde{\varphi}\left(\left\{\left(z_{1}, z_{2}, z_{3}\right), f\right\} / S L(2, C)\right)=f\left(z_{i}\right)
\end{aligned}
$$

where $H$ is hyperplane bundle on $C P^{N-1}, \tilde{\pi_{3}}$ is 3 -fold forgetful map from $\mathcal{M}_{0, d, 3}^{C P^{N-1}}$ to $\mathcal{M}_{0, d, 0}^{C P^{N-1}}$ and $\mathcal{E}_{k d+1}$ denotes direct image sheaf $R_{\pi_{1}}^{0}\left(\tilde{\varphi}_{1}^{*}(k H)\right)$ coming from forgetful map $\pi_{1}$ from $\mathcal{M}_{0, d, 1}^{C P^{N-1}}$ to $\mathcal{M}_{0, d, 0}^{C P^{N-1}}$.

2. We can consider $\mathcal{O}_{e}$ as the generator of $H_{q, e}^{*}\left(M_{N, k}\right)$, and we only have to determine multiplication rule for $\mathcal{O}_{e}$. In other words, if we set

$$
\begin{aligned}
F_{\alpha}^{N, k}: \mathcal{O}_{e} \cdot \mathcal{O}_{e^{\alpha}}= & \sum_{d=0}^{\left[\frac{1+\alpha}{N-k}\right]}\left\langle\mathcal{O}_{e} \mathcal{O}_{e^{\alpha}} \mathcal{O}_{e^{N-3-\alpha+(N-k) d}}\right\rangle \frac{1}{k} \mathcal{O}_{e^{1+\alpha-(N-k) d}} \\
(k<N) & \\
F_{\alpha}^{N, N}: \mathcal{O}_{e} \cdot \mathcal{O}_{e^{\alpha}}= & \left\langle\mathcal{O}_{e} \mathcal{O}_{e^{\alpha}} \mathcal{O}_{e^{N-3-\alpha}}\right\rangle \frac{1}{N} \mathcal{O}_{e^{1+\alpha}} \\
& (k=N)
\end{aligned}
$$


, $H_{q}^{*}\left(M_{N}^{k}\right)$ is constructed under the assumption of its commutativity and associativity as follows.

$$
C\left[\mathcal{O}_{e}, \mathcal{O}_{e^{2}}, \cdots, \mathcal{O}_{e^{N-2}}\right] / I\left[F_{1}^{N, k}, F_{2}^{N, k}, \cdots, F_{N-2}^{N, k}\right]
$$

where $C\left[\mathcal{O}_{e}, \mathcal{O}_{e^{2}}, \cdots, \mathcal{O}_{e^{N-2}}\right]$ denotes the polynomial ring generated by $\mathcal{O}_{e^{\alpha}}$ and $I\left[F_{1}^{N, k}, F_{2}^{N, k}, \cdots, F_{N-2}^{N, k}\right]$ is the ideal generated by $F_{\alpha}^{N, k}$ 's.

We calculate $F_{\alpha}^{N, k}$ for $k \leq N-2$ and $N \leq 9$ case and find the ideal includes the following relation.

$$
\left(\mathcal{O}_{e}\right)^{N-1}-k^{k} \cdot q \cdot\left(\mathcal{O}_{e}\right)^{k-1}=0
$$

Numerical results are shown in appendix A. In this case, using (63) and $\left\langle\prod_{i=1}^{N-2} \mathcal{O}_{e}\left(z_{i}\right)\right\rangle_{0}=k$, we can easily see from $(50)$

$$
\left\langle\prod_{i=1}^{N-2+(N-k) d} \mathcal{O}_{e}\left(z_{i}\right)\right\rangle=k^{k d+1} \cdot q^{d} .
$$

\section{Reformulation as One Variable Polynomial Al- gebra}

With some algebra, we can rewrite the relations (62) into the form

$$
\begin{gathered}
G_{\alpha}^{N, k}: \mathcal{O}_{e^{\alpha}}=\left(\mathcal{O}_{e}\right)^{\alpha}-\sum_{d=1}^{\left[\frac{\alpha}{N-k}\right]} \gamma_{\alpha, d}^{N, k}\left(\mathcal{O}_{e}\right)^{\alpha-(N-k) d} \cdot q^{d} \\
(2 \leq \alpha \leq N-2) \\
G_{r e l}^{N, k}: 0=\left(\mathcal{O}_{e}\right)^{N-1}-\sum_{d=1}^{\left[\frac{N-1}{N-k}\right]} \delta_{d}^{N, k}\left(\mathcal{O}_{e}\right)^{N-1-(N-k) d} \cdot q^{d} \\
(N>k) \quad\left(\prod_{j=1}^{\alpha} \gamma_{j}^{N, N}(q)\right)\left(\mathcal{O}_{e}\right)^{\alpha} \\
G_{\alpha}^{N, N}: \mathcal{O}_{e^{\alpha}}={ }^{(2 \leq \alpha \leq N-2)} \\
G_{r e l}^{N, N}: 0=\quad\left(\prod_{j=1}^{N-2} \gamma_{j}^{N, N}(q)\right)\left(\mathcal{O}_{e}\right)^{N-1} \\
(N=k)
\end{gathered}
$$

where

$$
\gamma_{j}^{N, N}(q):=N /\left\langle\mathcal{O}_{e} \mathcal{O}_{e^{j-1}} \mathcal{O}_{e^{N-2-j}}\right\rangle
$$


Then we can realize $H_{q, e}^{*}\left(M_{N}^{k}\right)$ as one variable polynomial algebra by regarding $\mathcal{O}_{e^{0}}$ as $1, \mathcal{O}_{e}$ as $X, \mathcal{O}_{e^{\alpha}}$ as r.h.s of $G_{\alpha}^{N, k}$, and $G_{r e l}^{N, k}$ as a relation. And if we set

$$
\begin{aligned}
& f_{0}^{N, k}(X):=1, f_{1}^{N, k}(X):=X \\
& f_{\alpha}^{N, k}(X):=\text { r.h.s. of } G_{\alpha}^{N, k} \quad(\alpha=2, \cdots, N-2) \\
& f_{r e l .}^{N, k}(X):=\text { r.h.s. of } G_{r e l}^{N, k},
\end{aligned}
$$

correlation functions are written in the residue form which follows from (53) as is well-known in [4],

$$
\left\langle\mathcal{O}_{e^{\alpha^{1}}} \mathcal{O}_{e^{\alpha^{2}}} \cdots \mathcal{O}_{e^{\alpha^{l}}}\right\rangle_{M_{N}^{k}}=k \cdot \oint_{C_{\infty}}\left(\frac{f_{\alpha_{1}}^{N, k}(X) \cdot f_{\alpha_{2}}^{N, k}(X) \cdots f_{\alpha_{l}}^{N, k}(X)}{f_{r e l}^{N, k}(X)}\right) d X
$$

where the integration contour $C_{\infty}$ is a small circle around $X=\infty$ in the counterclockwise direction. These results are collected in Appendix B.

\section{Proof of (72)}

First, we assume the following relation,

$$
f_{\alpha_{1}}^{N, k}(X) \cdot f_{\alpha_{2}}^{N, k}(X)=h(X) f_{r e l}^{N, k}(X)+\left\langle\mathcal{O}_{e^{\alpha_{1}}} \mathcal{O}_{e^{\alpha_{2}}} \mathcal{O}_{e^{h}}\right\rangle \eta^{h m} f_{m}^{N, k}(X)
$$

where $h(X)$ is a certain polynomial of $X$. To be more precise, we have to "check" (73) by numerical calculation to justify our construction but we heuristically rely on completeness of this algebra. Then

$$
\begin{aligned}
\text { (r.h.s) of }(72) & =k \cdot \oint_{C_{\infty}} h(X) \cdot\left(f_{\alpha_{3}}^{N, k}(X) \cdot f_{\alpha_{4}}^{N, k}(X) \cdots f_{\alpha_{l}}^{N, k}(X)\right) d X \\
& +\left\langle\mathcal{O}_{e^{\alpha_{1}}} \mathcal{O}_{e^{\alpha_{2}}} \mathcal{O}_{e^{h}}\right\rangle \eta^{h m} k \cdot \oint_{C_{\infty}}\left(\frac{f_{m}^{N, k}(X) \cdot f_{\alpha_{3}}^{N, k}(X) \cdots f_{\alpha_{l}}^{N, k}(X)}{f_{r e l}^{N, k}(X)}\right) d X
\end{aligned}
$$

The first term of (74) vanishes because if we set $X^{\prime}=\frac{1}{X}$, we have terms with degree no more than -2 in the integrand. And we have shown fusion rule for representation of $(72)$.

Then by induction, we only have to show the following formula.

$$
k \cdot \oint_{C_{\infty}} \frac{f_{\alpha_{1}}^{N, k}(X) \cdot f_{\alpha_{2}}^{N, k}(X)}{f_{r e l}^{N, k}(X)}=k \cdot \delta_{\alpha_{1}+\alpha_{2}, N-2}
$$

But, by inserting $\mathcal{O}_{e^{0}}=1$ formally, (this insertion does not change the value of correlation function) and using (73) again, we only have to show the following relation instead of (75).

$$
k \cdot \oint_{C_{\infty}} \frac{f_{\alpha}^{N, k}(X)}{f_{r e l}^{N, k}(X)}=k \cdot \delta_{\alpha, N-2}
$$

(76) is trivial if we change the variable $X$ into $X^{\prime}=\frac{1}{X}$. 
At first sight, this reformulation seems to be superficial, but we find some curious relation between $\gamma_{\alpha, d}^{N, k}$ for $k \leq N-2$ case.

relation 1

$$
\gamma_{\alpha, 1}^{N, k}=\gamma_{\alpha-1,1}^{N-1, k} \quad(k \leq N-2)
$$

relation 2

$$
\begin{aligned}
& \gamma_{N-2,2}^{N, k}=\frac{\left(\gamma_{N-k, 1}^{N, k}\right)^{2}}{2} \\
& ((N-k) 2=N-2) \\
& \gamma_{N-3,2}^{N, k}=\gamma_{N-k, 1}^{N, k}\left(\frac{\gamma_{N-k+1,1}^{N, k}}{2}-\frac{\gamma_{N-k, 1}^{N, k}}{4}\right) \\
& \gamma_{N-2,2}^{N, k}=\gamma_{N-k, 1}^{N, k}\left(\frac{\gamma_{N-k+1,1}^{N, k}}{2}+\frac{\gamma_{N-k, 1}^{N, k}}{4}\right) \\
& ((N-k) 2=N-3) \\
& \gamma_{N-4,2}^{N, k}=\gamma_{N-k, 1}^{N, k}\left(\frac{\gamma_{N-k+2,1}^{N, k}}{2}-\frac{\gamma_{N-k+1,1}^{N, k}}{4}-\frac{\gamma_{N-k, 1}^{N, k}}{8}\right) \\
& \gamma_{N-3,2}^{N, k}=\frac{\left(\gamma_{N-k+1,1}^{N, k}\right)^{2}}{2} \\
& \gamma_{N-2,2}^{N, k}=\gamma_{N-k, 1}^{N, k}\left(\frac{\gamma_{N-k+2,1}^{N, k}}{2}+\frac{\gamma_{N-k+1,1}^{N, k}}{4}+\frac{\gamma_{N-k, 1}^{N, k}}{8}\right) \\
& ((N-k) 2=N-4) \\
& \gamma_{N-5,2}^{N, k}=\gamma_{N-k, 1}^{N, k}\left(\frac{\gamma_{N-k+3,1}^{N, k}}{2}-\frac{\gamma_{N-k+2,1}^{N, k}}{4}-\frac{\gamma_{N-k+1,1}^{N, k}}{8}-\frac{\gamma_{N-k, 1}^{N, k}}{16}\right) \\
& \gamma_{N-4,2}^{N, k}=\gamma_{N-k+1,1}^{N, k}\left(\frac{\gamma_{N-k+2,1}^{N, k}}{2}-\frac{\gamma_{N-k+1,1}^{N, k}}{4}\right) \\
& +\frac{\gamma_{N-k, 1}^{N, k}}{2}\left(\frac{\gamma_{N-k+2,1}^{N, k}}{2}-\frac{\gamma_{N-k+1,1}^{N, k}}{4}-\frac{\gamma_{N-k, 1}^{N, k}}{8}\right) \\
& \gamma_{N-3,2}^{N, k}=\gamma_{N-k+1,1}^{N, k}\left(\frac{\gamma_{N-k+2,1}^{N, k}}{2}+\frac{\gamma_{N-k+1,1}^{N, k}}{4}\right) \\
& -\frac{\gamma_{N-k, 1}^{N, k}}{2}\left(\frac{\gamma_{N-k+2,1}^{N, k}}{2}-\frac{\gamma_{N-k+1,1}^{N, k}}{4}-\frac{\gamma_{N-k, 1}^{N, k}}{8}\right) \\
& \gamma_{N-2,2}^{N, k}=\gamma_{N-k, 1}^{N, k}\left(\frac{\gamma_{N-k+3,1}^{N, k}}{2}+\frac{\gamma_{N-k+2,1}^{N, k}}{4}+\frac{\gamma_{N-k+1,1}^{N, k}}{8}+\frac{\gamma_{N-k, 1}^{N, k}}{16}\right) \\
& ((N-k) 2=N-5)
\end{aligned}
$$

relation 3

$$
\begin{aligned}
\gamma_{N-2,3}^{N, k}= & \gamma_{N-k, 1}^{N, k} \gamma_{2(N-k), 2}^{N, k} \\
& ((N-k) 3=N-2)
\end{aligned}
$$

We can reconstruct some of the above relations from the compatibility of the expansion form of (37) and relation (63), but we are not sure that all of them follow from 
it at this stage. With these relations, we can figure out some characteristic feature of $H_{q, e}^{*}\left(M_{N}^{k}\right)$.

First, quantum correction of degree 1 to $H_{q, e}^{*}\left(M_{N}^{k}\right)$ does not depend on $N$, which can be easily seen from relation 1 . So we think these correction coefficients $\gamma_{\alpha, 1}^{k}(:=$ $\gamma_{N-k+\alpha-1,1}^{N, k}$ ) play central role in the ring when $k \leq N-2$. In other words, we expect all the higher degree quantum correction coefficients are determined by $\gamma_{\alpha, 1}^{k}$. Relation 2 are found from these speculations. Second, from the expansion form of (66), degree $\mathrm{d}$ coefficients of $\mathcal{O}_{e^{\alpha}}$ occur when $\alpha \geq(N-k) d$ holds. Then if $k \leq\left[\frac{N}{2}\right]+1$, no corrections occur from sectors with degree greater than 1 . But degree 1 corrections remain stable since they exist as long as $\alpha$ is no less than $N-k$. This seems to support our first speculation. We will show some examples of these features using the results of $H_{q, e}^{*}\left(M_{N}^{6}\right)$.

$$
\begin{aligned}
& H_{q, e}^{*}\left(M_{8}^{6}\right) \\
& \mathcal{O}_{e}=X \\
& \mathcal{O}_{e^{2}}=X^{2}-\gamma_{1,1}^{6} q \\
& \mathcal{O}_{e^{3}}=X^{3}-\gamma_{2,1}^{6} X q \\
& \mathcal{O}_{e^{4}}=X^{4}-\gamma_{3,1}^{6} X^{2} q-\gamma_{1,1}^{6}\left(\frac{\gamma_{3,1}^{6}}{2}-\frac{\gamma_{2,1}^{6}}{4}-\frac{\gamma_{1,1}^{6}}{8}\right) q^{2} \\
& \mathcal{O}_{e^{5}}=X^{5}-\gamma_{4,1}^{6} X^{3} q-\frac{\left(\gamma_{2,1}^{6}\right)^{2}}{2} X q^{2} \\
& \mathcal{O}_{e^{6}}=X^{6}-\gamma_{5,1}^{6} X^{4} q-\gamma_{1,1}^{6}\left(\frac{\gamma_{3,1}^{6}}{2}+\frac{\gamma_{2,1}^{6}}{4}+\frac{\gamma_{1,1}^{6}}{8}\right) X^{2} q^{2} \\
& -\left(\gamma_{1,1}^{6}\right)^{2}\left(\frac{\gamma_{3,1}^{6}}{2}-\frac{\gamma_{2,1}^{6}}{4}-\frac{\gamma_{1,1}^{6}}{8}\right) q^{3} \\
& H_{q, e}^{*}\left(M_{9}^{6}\right) \\
& \mathcal{O}_{e}=X \\
& \mathcal{O}_{e^{2}}=X^{2} \\
& \mathcal{O}_{e^{3}}=X^{3}-\gamma_{1,1}^{6} q \\
& \mathcal{O}_{e^{4}}=X^{4}-\gamma_{2,1}^{6} X^{1} q \\
& \mathcal{O}_{e^{5}}=X^{5}-\gamma_{3,1}^{6} X^{2} q \\
& \mathcal{O}_{e^{6}}=X^{6}-\gamma_{4,1}^{6} X^{3} q-\gamma_{1,1}^{6}\left(\frac{\gamma_{2,1}^{6}}{2}-\frac{\gamma_{1,1}^{6}}{4}\right) q^{2} \\
& \mathcal{O}_{e^{7}}=X^{7}-\gamma_{5,1}^{6} X^{4} q-\gamma_{1,1}^{6}\left(\frac{\gamma_{2,1}^{6}}{2}+\frac{\gamma_{1,1}^{6}}{4}\right) X q^{2} \\
& H_{q, e}^{*}\left(M_{10}^{6}\right) \\
& \mathcal{O}_{e}=X \\
& \mathcal{O}_{e^{2}}=X^{2} \\
& \mathcal{O}_{e^{3}}=X^{3}
\end{aligned}
$$




$$
\begin{aligned}
\mathcal{O}_{e^{4}} & =X^{4}-\gamma_{1,1}^{6} q \\
\mathcal{O}_{e^{5}} & =X^{5}-\gamma_{2,1}^{6} X q \\
\mathcal{O}_{e^{6}} & =X^{6}-\gamma_{3,1}^{6} X^{2} q \\
\mathcal{O}_{e^{7}} & =X^{7}-\gamma_{4,1}^{6} X^{3} q \\
\mathcal{O}_{e^{8}} & =X^{8}-\gamma_{5,1}^{6} X^{4} q-\frac{\left(\gamma_{1,1}^{6}\right)^{2}}{2} q^{2} \\
H_{q, e}^{*}\left(M_{N}^{6}\right) & \quad(N \geq 11) \\
\mathcal{O}_{e^{k}} & =X^{k} \quad(1 \leq k \leq N-7) \\
\mathcal{O}_{e^{N-7+\alpha}} & =X^{N-7+\alpha}-\gamma_{\alpha, 1}^{6} X^{\alpha-1} q(1 \leq \alpha \leq 5)
\end{aligned}
$$

where

$$
\gamma_{1,1}^{6}=720, \gamma_{2,1}^{6}=6984, \gamma_{3,1}^{6}=23328, \gamma_{4,1}^{6}=39672, \gamma_{5,1}^{6}=45936
$$

\section{Geometrical Interpretation}

In this section, we will briefly discuss why relation (63) or equation (64) holds from the geometrical point of view. Since $M_{N}^{k}$ is hypersurface in $C P^{N-1}, \mathcal{M}_{N, k,(\text { matter })}^{d}$ is submanifold of $\mathcal{M}_{C P^{N-1},(\text { matter })}^{d}$. So we can expect the following formula.

$$
\left\langle\prod_{i=1}^{N-2+(N-k) d} \mathcal{O}_{e}\left(z_{i}\right)\right\rangle=\int_{\mathcal{M}_{0, d}^{M_{N}^{k}}} \bigwedge_{i=1}^{N-2+(N-k) d} \phi_{i}^{*}(e)=\int_{\mathcal{M}_{0, d}^{C P^{N-1}}} c_{(N, k)} \wedge\left(\bigwedge_{i=1}^{N-2+(N-k) d} \varphi_{i}^{*}(e)\right)
$$

where

$$
\begin{aligned}
\phi_{i}: \mathcal{M}_{0, d}^{M_{N}^{k}} & \mapsto M_{N}^{k}, \phi_{i}(f)=f\left(z_{i}\right) \\
\varphi_{i}: \mathcal{M}_{0, d}^{C P^{N-1}} & \mapsto C P^{N-1}, \quad \varphi_{i}(f)=f\left(z_{i}\right)
\end{aligned}
$$

$c_{(N, k)}$ is the form which impose the following condition on $f \in \mathcal{M}_{0, d}^{C P^{N-1}}$.

$$
f\left(C P^{1}\right) \subset M_{N}^{k}
$$

Since holomorphic map from $C P^{1}$ to $C P^{N-1}$ of degree d is described by the polynomial map as follows, we can roughly compactify $\mathcal{M}_{0, d}^{C P^{N-1}}$ into $C P^{N(d+1)-1}$.

$$
\begin{aligned}
& (s: t) \mapsto\left(\sum_{j=0}^{d} a_{j}^{1} s^{j} t^{d-j}: \sum_{j=0}^{d} a_{j}^{2} s^{j} t^{d-j}: \cdots: \sum_{j=0}^{d} a_{j}^{N} s^{j} t^{d-j}\right) \\
& :=\left(A_{d}^{1}(s, t): A_{d}^{2}(s, t): \cdots: A_{d}^{N}(s, t)\right) \\
& \overline{\mathcal{M}}_{0, d}^{C P^{N-1}}=\left(a_{0}^{1}: a_{0}^{1}: \cdots: a_{d}^{1}: \cdots: \cdots: a_{0}^{N}: \cdots: a_{d}^{N}\right)
\end{aligned}
$$


Using this compactification, we can realize $c_{(N, k)}$ as follows.

$$
\begin{aligned}
f\left(C P^{1}\right) \subset M_{N}^{k} & \\
& \Longleftrightarrow \sum_{i=1}^{N}\left(\sum_{j=0}^{d} a_{j}^{i} s^{j} t^{d-j}\right)^{k}=0 \quad \text { for all } \quad(s, t) \\
& \Longleftrightarrow \sum_{l=1}^{k d} f_{l}\left(a_{j}^{i}\right) s^{k d-l} t^{l}=0 \quad \text { for all } \quad(s, t) \\
& \Longleftrightarrow f_{l}\left(a_{j}^{i}\right)=0 \quad l=0,1, \cdots, k d+1
\end{aligned}
$$

Since each $f_{l}\left(a_{j}^{i}\right)$ is a homogeneous polynomial of $a_{j}^{i}$ of degree $k$, we can regard them as $k \tilde{e}:=k c_{1}(\tilde{H})$ where $\tilde{H}$ is hyperplane bundle of $C P^{N(d+1)-1}$. And we have

$$
c_{(N, k)}=(k \tilde{e})^{k d+1}
$$

We can easily see that $\varphi_{i}^{*}(e)=\tilde{e}$ from the definition of $\varphi_{i}$ and we have

$$
\begin{aligned}
\left\langle\prod_{i=1}^{N-2+(N-k) d} \mathcal{O}_{e}\left(z_{i}\right)\right\rangle_{c} & =\int_{C P^{N(d+1)-1}}(k \tilde{e})^{k d+1} \wedge(\tilde{e})^{N-2+(N-k) d} \\
& =k^{k d+1}
\end{aligned}
$$

Our calculation in section 3 tells us this compactification gives an exact result when $k \leq N-2$. To derive this bound geometrically, we have to analyze points in $C P^{N(d+1)-1}$ added in the process of compactification. As was said in [21] and [28], these points are characterized by the fact that all $A_{d}^{i}$ 's have common divisor. This situation can be described by the following sequence of maps.

$$
\begin{aligned}
& C P^{N(d+1)-1} \stackrel{\eta_{1}}{\longleftarrow} \\
& C P^{N(d)-1} \times C P^{1} \stackrel{\eta_{2}}{\longleftarrow} C P^{N(d-1)-1} \times\left(C P^{1}\right)^{2} \stackrel{\eta_{3}}{\longleftarrow} \\
& \\
& \cdots \\
& C P^{N(d-k+1)-1} \times\left(C P^{1}\right)^{k} \stackrel{\eta_{k+1}}{\longleftarrow} C P^{N(d-k)-1} \times\left(C P^{1}\right)^{k+1} \stackrel{\eta_{k+2}}{\longleftarrow} \\
& \quad \cdots \\
& \\
& C P^{2 N-1} \times\left(C P^{1}\right)^{d-1} \stackrel{\eta_{d}}{\longleftarrow} C P^{N-1} \times\left(C P^{1}\right)^{d}
\end{aligned}
$$

where

$$
\begin{aligned}
\eta_{j}: & C P^{N(d-j+1)-1} \times\left(C P^{1}\right)^{j} \mapsto C P^{N(d-j+2)-1} \times\left(C P^{1}\right)^{j-1} \\
& \left(\left(A_{d-j}^{1}(s, t), \cdots, A_{d-j}^{N}(s, t)\right),\left(a^{1} s+b^{1} t\right), \cdots,\left(a^{j} s+b^{j} t\right)\right) \\
& \mapsto\left(\left(A_{d-j}^{1}(s, t)\left(a^{1} s+b^{1} t\right), \cdots, A_{d-j}^{N}(s, t)\left(a^{1} s+b^{1} t\right)\right)\right. \\
& \left.,\left(a^{2} s+b^{2} t\right), \cdots,\left(a^{j} s+b^{j} t\right)\right)
\end{aligned}
$$

In the calculation, we must treat $\operatorname{Im}\left(\eta_{j}\right)$ carefully. Then why (102) is correct in $k \leq N-2$ case ? This can be understood as follows. Consider the first non-trivial boundary $\operatorname{Im}\left(\eta_{1}\right)$.

$$
\eta_{1}\left(C P^{N d-1} \times C P^{1}\right)=\left((a s+b t) A_{d-1}^{1}(s, t), \cdots,(a s+b t) A_{d-1}^{N}(s, t)\right)
$$


For these points, the condition $f\left(C P^{1}\right) \subset M_{N}^{k}$ acts only on $A_{d-1}^{i}(s, t)^{\prime}$ 's and the remaining degrees of freedom come from $C P^{1}$ and constrained $A_{d-1}^{i}(s, t)$ 's, i.e.

$$
\begin{aligned}
\sharp(\text { degrees of freedom }) & =1+N d-1-(k(d-1)+1) \\
& =(N-k) d+k-1
\end{aligned}
$$

Then if the condition $k \leq N-2$ is satisfied, we have

$$
\begin{aligned}
(N-k) d+k-1 & <(N-k) d+N-2 \\
& =\operatorname{dim}\left(\mathcal{M}_{0, d}^{M_{N}^{k}}\right)
\end{aligned}
$$

(106) tells us that the condition (100) may make the contribution from $\operatorname{Im}\left(\eta_{1}\right)$ be a space whose dimension is no less than $\operatorname{dim}\left(\mathcal{M}_{0, d}^{M_{N}^{k}}\right)$. But in $k \leq N-2$ case, (107) assures us that it is irrelevant in view of the dimensional counting.

\section{Conclusion}

Our main result of this paper is the determination of the bound $k \leq N-2$. Under this bound, principal relation of quantum cohomology ring is written in a simple form, $\left(\mathcal{O}_{e}\right)^{N-1}=k^{k} q\left(\mathcal{O}_{e}\right)^{k-1}$, which is a natural generalization of the well-known result of $C P^{N-2}$ model, $\left(\mathcal{O}_{e}\right)^{N-1}=q$ [20]. The ring $H_{q, e}^{*}\left(M_{N}^{k}\right)$ is mainly characterized by $k$, so polynomial representations of operators with different $N$ are alike with each other. These seem to be determined by the correction coefficients $\gamma_{\alpha, 1}^{k}$ coming from holomorphic maps of degree 1 which are invariant under variation of $N$, though we cannot give complete formulation in this paper.

We give geometrical interpretation of this bound in section 5 but this argument does not explain why insertion of operator $\mathcal{O}_{e^{2}}$ and $\left(\mathcal{O}_{e}\right)^{2}$ are distinct even if $k \leq N-2$. These insertions cannot be distinguished by our simple logic. Looking around the situations, it seems to be effective only with the insertion of BRST-closed operator induced from Kähler forms in treating hypersurface in weighted projective space. Of course, as can be seen in [2], moduli spaces of manifolds like Grassmannians which are defined as homogeneous spaces are compactified without ambiguous process like the one in $(100)$. In this case, such troubles do not arise.

Finally, we discuss what our results tells us with respect to $k=N-1, N$ case. At least, it supports our assertion that the first term of $N$-expansion of $\left\langle\prod_{i=1}^{N-2} \mathcal{O}_{e}\left(z_{i}\right)\right\rangle$ on Calabi-Yau manifold $M_{N}^{N}$ comes from the compactifiaction treated in this paper but explanation of correction terms from this point of view is still not clear.

Acknowledgment I'd like to thank Dr.K.Hori and Prof.T.Eguchi for many useful discussions. I also thank Dr.Y.Sun and Dr.M.Nagura for kind encouragement. 


\section{Appendix A Multiplication Rules of $H_{q, e}^{*}\left(M_{N}^{k}\right)$}

$$
\begin{aligned}
& H_{q, e}^{*}\left(M_{N}^{1}\right) \\
& \mathcal{O}_{e} \cdot \mathcal{O}_{e^{\alpha}}=\mathcal{O}_{e^{\alpha+1}} \quad(0 \leq \alpha \leq N-3) \quad \mathcal{O}_{e} \cdot \mathcal{O}_{e^{N-2}}=q \\
& H_{q, e}^{*}\left(M_{N}^{2}\right) \\
& \mathcal{O}_{e} \cdot \mathcal{O}_{e^{\alpha}}=\mathcal{O}_{e^{\alpha+1}} \quad(0 \leq \alpha \leq N-4) \quad \mathcal{O}_{e} \cdot \mathcal{O}_{e^{N-3}}=\mathcal{O}_{e^{N-2}}+2 q \\
& \mathcal{O}_{e} \cdot \mathcal{O}_{e^{N-2}}=2 \mathcal{O}_{e} q \\
& H_{q, e}^{*}\left(M_{5}^{3}\right) \\
& \mathcal{O}_{e} \cdot \mathcal{O}_{e}=\mathcal{O}_{e^{2}}+6 q \quad \mathcal{O}_{e} \cdot \mathcal{O}_{e^{2}}=\mathcal{O}_{e^{3}}+15 \mathcal{O}_{e} q \\
& \mathcal{O}_{e} \cdot \mathcal{O}_{e^{3}}=6 \mathcal{O}_{e^{2}} q+36 q^{2} \\
& H_{q, e}^{*}\left(M_{N}^{3}\right) \quad(N \geq 6) \\
& \mathcal{O}_{e} \cdot \mathcal{O}_{e^{\alpha}}=\mathcal{O}_{e^{\alpha+1}} \quad(0 \leq \alpha \leq N-5) \\
& \mathcal{O}_{e} \cdot \mathcal{O}_{e^{N-4}}=\mathcal{O}_{e^{N-3}}+6 q \quad \mathcal{O}_{e} \cdot \mathcal{O}_{e^{N-3}}=\mathcal{O}_{e^{N-2}}+15 \mathcal{O}_{e} q \\
& \mathcal{O}_{e} \cdot \mathcal{O}_{e^{N-2}}=6 \mathcal{O}_{e^{2}} q \\
& H_{q, e}^{*}\left(M_{6}^{4}\right) \\
& \mathcal{O}_{e} \cdot \mathcal{O}_{e}=\mathcal{O}_{e^{2}}+24 q \\
& \mathcal{O}_{e} \cdot \mathcal{O}_{e^{2}}=\mathcal{O}_{e^{3}}+104 \mathcal{O}_{e} q \\
& \mathcal{O}_{e} \cdot \mathcal{O}_{e^{3}}=\mathcal{O}_{e^{4}}+104 \mathcal{O}_{e^{2}} q+2784 q^{2} \\
& \mathcal{O}_{e} \cdot \mathcal{O}_{e^{4}}=24 \mathcal{O}_{e^{3}} q+2784 \mathcal{O}_{e} q^{2} \\
& H_{q, e}^{*}\left(M_{7}^{4}\right) \\
& \mathcal{O}_{e} \cdot \mathcal{O}_{e}=\mathcal{O}_{e^{2}} \\
& \mathcal{O}_{e} \cdot \mathcal{O}_{e^{2}}=\mathcal{O}_{e^{3}}+24 q \\
& \mathcal{O}_{e} \cdot \mathcal{O}_{e^{3}}=\mathcal{O}_{e^{4}}+104 \mathcal{O}_{e} q \\
& \mathcal{O}_{e} \cdot \mathcal{O}_{e^{4}}=\mathcal{O}_{e^{5}}+104 \mathcal{O}_{e^{2}} q \\
& \mathcal{O}_{e} \cdot \mathcal{O}_{e^{5}}=24 \mathcal{O}_{e^{3}} q+576 q^{2} \\
& H_{q, e}^{*}\left(M_{N}^{4}\right) \\
& \mathcal{O}_{e} \cdot \mathcal{O}_{e^{\alpha}}=\mathcal{O}_{e^{\alpha+1}} \quad(0 \leq \alpha \leq N-6) \\
& \mathcal{O}_{e} \cdot \mathcal{O}_{e^{N-5}}=\mathcal{O}_{e^{N-4}}+24 q \\
& \mathcal{O}_{e} \cdot \mathcal{O}_{e^{N-4}}=\mathcal{O}_{e^{N-3}}+104 \mathcal{O}_{e} q \\
& \mathcal{O}_{e} \cdot \mathcal{O}_{e^{N-3}}=\mathcal{O}_{e^{N-2}}+104 \mathcal{O}_{e^{2}} q \\
& \mathcal{O}_{e} \cdot \mathcal{O}_{e^{N-2}}=24 \mathcal{O}_{e^{3}} q
\end{aligned}
$$




$$
\begin{aligned}
H_{q, e}^{*} & \left(M_{7}^{5}\right) \\
& \mathcal{O}_{e} \cdot \mathcal{O}_{e}=\mathcal{O}_{e^{2}}+120 q \\
& \mathcal{O}_{e} \cdot \mathcal{O}_{e^{2}}=\mathcal{O}_{e^{3}}+770 \mathcal{O}_{e} q \\
& \mathcal{O}_{e} \cdot \mathcal{O}_{e^{3}}=\mathcal{O}_{e^{4}}+1345 \mathcal{O}_{e^{2}} q+211200 q^{2} \\
& \mathcal{O}_{e} \cdot \mathcal{O}_{e^{4}}=\mathcal{O}_{e^{5}}+770 \mathcal{O}_{e^{3}} q+692500 \mathcal{O}_{e} q^{2} \\
& \mathcal{O}_{e} \cdot \mathcal{O}_{e^{5}}=120 \mathcal{O}_{e^{4}} q+211200 \mathcal{O}_{e^{2}} q^{2}
\end{aligned}
$$

$$
\begin{aligned}
H_{q, e}^{*}\left(M_{8}^{5}\right) & \\
\mathcal{O}_{e} \cdot \mathcal{O}_{e} & =\mathcal{O}_{e^{2}} \\
\mathcal{O}_{e} \cdot \mathcal{O}_{e^{2}} & =\mathcal{O}_{e^{3}}+120 q \\
\mathcal{O}_{e} \cdot \mathcal{O}_{e^{3}} & =\mathcal{O}_{e^{4}}+770 \mathcal{O}_{e} q \\
\mathcal{O}_{e} \cdot \mathcal{O}_{e^{4}} & =\mathcal{O}_{e^{5}}+1345 \mathcal{O}_{e^{2}} q \\
\mathcal{O}_{e} \cdot \mathcal{O}_{e^{5}} & =\mathcal{O}_{e^{6}}+770 \mathcal{O}_{e^{3}} q+99600 q^{2} \\
\mathcal{O}_{e} \cdot \mathcal{O}_{e^{6}} & =120 \mathcal{O}_{e^{4}} q+99600 \mathcal{O}_{e} q^{2}
\end{aligned}
$$

$$
\begin{aligned}
& H_{q, e}^{*}\left(M_{9}^{5}\right) \\
& \mathcal{O}_{e} \cdot \mathcal{O}_{e}=\mathcal{O}_{e^{2}} \\
& \mathcal{O}_{e} \cdot \mathcal{O}_{e^{2}}=\mathcal{O}_{e^{3}} \\
& \mathcal{O}_{e} \cdot \mathcal{O}_{e^{3}}=\mathcal{O}_{e^{4}}+120 q \\
& \mathcal{O}_{e} \cdot \mathcal{O}_{e^{4}}=\mathcal{O}_{e^{5}}+770 \mathcal{O}_{e} q \\
& \mathcal{O}_{e} \cdot \mathcal{O}_{e^{5}}=\mathcal{O}_{e^{6}}+1345 \mathcal{O}_{e^{2}} q \\
& \mathcal{O}_{e} \cdot \mathcal{O}_{e^{6}}=\mathcal{O}_{e^{7}}+770 \mathcal{O}_{e^{3}} q \\
& \mathcal{O}_{e} \cdot \mathcal{O}_{e^{7}}=+120 \mathcal{O}_{e^{4}} q+14400 q^{2}
\end{aligned}
$$

$$
\begin{aligned}
H_{q, e}^{*}\left(M_{N}^{5}\right) \quad(N \geq 10) & \\
\mathcal{O}_{e} \cdot \mathcal{O}_{e^{\alpha}} & =\mathcal{O}_{e^{\alpha+1}} \quad(0 \leq \alpha \leq N-7) \\
\mathcal{O}_{e} \cdot \mathcal{O}_{e^{N-6}} & =\mathcal{O}_{e^{N-5}}+120 q \\
\mathcal{O}_{e} \cdot \mathcal{O}_{e^{N-5}} & =\mathcal{O}_{e^{N-4}}+770 \mathcal{O}_{e} q \\
\mathcal{O}_{e} \cdot \mathcal{O}_{e^{N-4}} & =\mathcal{O}_{e^{N-3}}+1345 \mathcal{O}_{e^{2}} q \\
\mathcal{O}_{e} \cdot \mathcal{O}_{e^{N-3}} & =\mathcal{O}_{e^{N-2}}+770 \mathcal{O}_{e^{3}} q \\
\mathcal{O}_{e} \cdot \mathcal{O}_{e^{N-2}} & =120 \mathcal{O}_{e^{4}} q
\end{aligned}
$$




$$
\begin{aligned}
H_{q, e}^{*}\left(M_{8}^{6}\right) & \\
\mathcal{O}_{e} \cdot \mathcal{O}_{e}= & \mathcal{O}_{e^{2}}+720 q \\
\mathcal{O}_{e} \cdot \mathcal{O}_{e^{2}}= & \mathcal{O}_{e^{3}}+6264 \mathcal{O}_{e} q \\
\mathcal{O}_{e} \cdot \mathcal{O}_{e^{3}}= & \mathcal{O}_{e^{4}}+16344 \mathcal{O}_{e^{2}} q+18843840 q^{2} \\
\mathcal{O}_{e} \cdot \mathcal{O}_{e^{4}}= & \mathcal{O}_{e^{5}}+16344 \mathcal{O}_{e^{3}} q+131458464 \mathcal{O}_{e} q^{2} \\
\mathcal{O}_{e} \cdot \mathcal{O}_{e^{5}}= & \mathcal{O}_{e^{6}}+6264 \mathcal{O}_{e^{4}} q+131458464 \mathcal{O}_{e^{2}} q^{2} \\
& +144069995520 q^{3} \\
\mathcal{O}_{e} \cdot \mathcal{O}_{e^{6}}= & +720 \mathcal{O}_{e^{5}} q+18843840 \mathcal{O}_{e^{3}} q^{2} \\
& +14406999520 \mathcal{O}_{e} q^{3} \\
H_{q, e}^{*}\left(M_{9}^{6}\right)= & \\
\mathcal{O}_{e} \cdot \mathcal{O}_{e}= & \mathcal{O}_{e^{2}} \\
\mathcal{O}_{e} \cdot \mathcal{O}_{e^{2}}= & \mathcal{O}_{e^{3}}+720 q \\
\mathcal{O}_{e} \cdot \mathcal{O}_{e^{3}}= & \mathcal{O}_{e^{4}}+6264 \mathcal{O}_{e} q \\
\mathcal{O}_{e} \cdot \mathcal{O}_{e^{4}}= & \mathcal{O}_{e^{5}}+16344 \mathcal{O}_{e^{2}} q \\
\mathcal{O}_{e} \cdot \mathcal{O}_{e^{5}=}= & \mathcal{O}_{e^{6}}+16344 \mathcal{O}_{e^{3}} q+14152320 q^{2} \\
\mathcal{O}_{e} \cdot \mathcal{O}_{e^{6}=}= & \mathcal{O}_{e^{7}}+6264 \mathcal{O}_{e^{4}} q+44006976 \mathcal{O}_{e} q^{2} \\
\mathcal{O}_{e} \cdot \mathcal{O}_{e^{7}}= & 720 \mathcal{O}_{e^{5}} q+14152320 \mathcal{O}_{e^{2}} q^{2} \\
H_{q, e}^{*}\left(M_{10}^{6}\right) & \\
\mathcal{O}_{e} \cdot \mathcal{O}_{e}= & \mathcal{O}_{e^{2}} \\
\mathcal{O}_{e} \cdot \mathcal{O}_{e^{2}}= & \mathcal{O}_{e^{3}} \\
\mathcal{O}_{e} \cdot \mathcal{O}_{e^{3}}= & \mathcal{O}_{e^{4}}+720 q \\
\mathcal{O}_{e} \cdot \mathcal{O}_{e^{4}}= & \mathcal{O}_{e^{5}}+6264 \mathcal{O}_{e} q \\
\mathcal{O}_{e} \cdot \mathcal{O}_{e^{5}}= & \mathcal{O}_{e^{6}}+16344 \mathcal{O}_{e^{2}} q \\
\mathcal{O}_{e} \cdot \mathcal{O}_{e^{6}}= & \mathcal{O}_{e^{6}}+16344 \mathcal{O}_{e^{3}} q \\
\mathcal{O}_{e} \cdot \mathcal{O}_{e^{7}}= & \mathcal{O}_{e^{8}}+6264 \mathcal{O}_{e^{4}} q+4769280 \mathcal{O}_{e} q^{2} \\
\mathcal{O}_{e} \cdot \mathcal{O}_{e^{8}}= & 720 \mathcal{O}_{e^{5}} q+4769280 \mathcal{O}_{e^{2}} q^{2} \\
&
\end{aligned}
$$




$$
\begin{aligned}
H_{q, e}^{*}\left(M_{9}^{7}\right)= & \mathcal{O}_{e^{2}}+5040 q \\
\mathcal{O}_{e} \cdot \mathcal{O}_{e}= & \mathcal{O}_{e^{3}}+56196 \mathcal{O}_{e} q \\
\mathcal{O}_{e} \cdot \mathcal{O}_{e^{2}}= & \mathcal{O}_{e^{4}}+200452 \mathcal{O}_{e^{2}} q+205625920 q^{2} \\
\mathcal{O}_{e} \cdot \mathcal{O}_{e^{3}}= & \mathcal{O}_{e} \cdot \mathcal{O}_{e^{4}}= \\
\mathcal{O}_{e} \cdot \mathcal{O}_{e^{5}}= & \mathcal{O}_{e^{6}}+20046752 \mathcal{O}_{e^{3}} q+24699506832 \mathcal{O}_{e} q^{2} \\
& +534155202302400 q^{3} \\
\mathcal{O}_{e} \cdot \mathcal{O}_{e^{6}}= & \mathcal{O}_{e^{7}}+56196 \mathcal{O}_{e^{5}} q+2469950683252 \mathcal{O}_{e^{3}} q^{2} \\
& +1920365635990032 \mathcal{O}_{e} q^{3} \\
\mathcal{O}_{e} \cdot \mathcal{O}_{e^{7}}= & 5040 \mathcal{O}_{e^{6}} q+2056259520 \mathcal{O}_{e^{4}} q^{2} \\
& +534155202302400 \mathcal{O}_{e^{2}} q^{3} \\
& +5112982794486067200 q^{4}
\end{aligned}
$$

$$
\begin{aligned}
H_{q, e}^{*}\left(M_{10}^{7}\right) & \\
\mathcal{O}_{e} \cdot \mathcal{O}_{e}= & \mathcal{O}_{e^{2}} \\
\mathcal{O}_{e} \cdot \mathcal{O}_{e^{2}}= & \mathcal{O}_{e^{3}}+5040 q \\
\mathcal{O}_{e} \cdot \mathcal{O}_{e^{3}}= & \mathcal{O}_{e^{4}}+56196 \mathcal{O}_{e} q \\
\mathcal{O}_{e} \cdot \mathcal{O}_{e^{4}}= & \mathcal{O}_{e^{5}}+200452 \mathcal{O}_{e^{2}} q \\
\mathcal{O}_{e} \cdot \mathcal{O}_{e^{5}}= & \mathcal{O}_{e^{6}}+300167 \mathcal{O}_{e^{3}} q+2091962880 q^{2} \\
\mathcal{O}_{e} \cdot \mathcal{O}_{e^{6}}= & \mathcal{O}_{e^{7}}+200452 \mathcal{O}_{e^{4}} q+13570681320 \mathcal{O}_{e} q^{2} \\
\mathcal{O}_{e} \cdot \mathcal{O}_{e^{7}}= & \mathcal{O}_{e^{8}}+56196 \mathcal{O}_{e^{5}} q+13570681320 \mathcal{O}_{e^{2}} q^{2} \\
\mathcal{O}_{e} \cdot \mathcal{O}_{e^{8}}= & 5040 \mathcal{O}_{e^{6}} q+2091962880 \mathcal{O}_{e^{3}} q^{2} \\
& +13462263763200 q^{3}
\end{aligned}
$$




$$
\begin{aligned}
H_{q, e}^{*}\left(M_{11}^{8}\right) & \\
\mathcal{O}_{e} \cdot \mathcal{O}_{e}= & \mathcal{O}_{e^{2}} \\
\mathcal{O}_{e} \cdot \mathcal{O}_{e^{2}}= & \mathcal{O}_{e^{3}}+40320 q \\
\mathcal{O}_{e} \cdot \mathcal{O}_{e^{3}}= & \mathcal{O}_{e^{4}}+554112 \mathcal{O}_{e} q \\
\mathcal{O}_{e} \cdot \mathcal{O}_{e^{4}}= & \mathcal{O}_{e^{5}}+2552192 \mathcal{O}_{e^{2}} q \\
\mathcal{O}_{e} \cdot \mathcal{O}_{e^{5}=}= & \mathcal{O}_{e^{6}}+5241984 \mathcal{O}_{e^{3}} q+345655618560 q^{2} \\
\mathcal{O}_{e} \cdot \mathcal{O}_{e^{6}=}= & \mathcal{O}_{e^{7}}+5241984 \mathcal{O}_{e^{4}} q+3857214283776 \mathcal{O}_{e} q^{2} \\
\mathcal{O}_{e} \cdot \mathcal{O}_{e^{7}}= & \mathcal{O}_{e^{8}}+2552192 \mathcal{O}_{e^{5}} q+8150222448640 \mathcal{O}_{e^{2}} q^{2} \\
\mathcal{O}_{e} \cdot \mathcal{O}_{e^{8}}= & \mathcal{O}_{e^{9}}+554112 \mathcal{O}_{e^{6}} q+3857214283776 \mathcal{O}_{e^{3}} q^{2} \\
& +235354398279598080 q^{3} \\
\mathcal{O}_{e} \cdot \mathcal{O}_{e^{9}}= & 40320 \mathcal{O}_{e^{7}} q+345655618560 \mathcal{O}_{e^{4}} q^{2} \\
& +235354398279598080 q^{3} \mathcal{O}_{e}
\end{aligned}
$$




\section{Appendix B One Variable Polynomial Representa- tion of $H_{q, e}^{*}\left(M_{N}^{k}\right)$}

$$
\begin{aligned}
H_{q, e}^{*}\left(M_{N}^{1}\right) & \\
f_{r e l}(X) & =X^{N-1}-q \\
\mathcal{O}_{e^{\alpha}} & =X^{\alpha} \quad(0 \leq \alpha \leq N-2) \\
H_{, e}^{*}\left(M_{N}^{2}\right) & \\
f_{r e l}(X) & =X^{N-1}-2^{2} X q \\
\mathcal{O}_{e^{\alpha}} & =X^{\alpha} \quad(0 \leq \alpha \leq N-3) \\
\mathcal{O}_{e^{N-2}} & =X^{N-2}-2 q X \\
H_{q, e}^{*}\left(M_{N}^{3}\right) & \\
f_{r e l}(X) & =X^{N-1}-3^{3} X^{2} q \\
\mathcal{O}_{e^{\alpha}} & =X^{\alpha} \quad(0 \leq \alpha \leq N-4) \\
\mathcal{O}_{e^{N-3}} & =X^{N-3}-6 q \\
\mathcal{O}_{e^{N-2}} & =X^{N-2}-21 X q \\
H_{q, e}^{*}\left(M_{6}^{4}\right) & \\
f_{r e l}(X) & =X^{5}-4^{4} X^{3} q \\
\mathcal{O}_{e^{0}} & =1 \\
\mathcal{O}_{e} & =X \\
\mathcal{O}_{e^{2}} & =X^{2}-24 q \\
\mathcal{O}_{e^{3}} & =X^{3}-128 X q \\
\mathcal{O}_{e^{4}} & =X^{4}-232 X^{2} q-288 q^{2} \\
H_{q, e}^{*}\left(M_{N}^{4}\right)(N & \geq 7) \\
f_{r e l}(X) & =X^{N-1}-4^{4} X^{3} q \\
\mathcal{O}_{e^{\alpha}} & =X^{\alpha} \quad(0 \leq \alpha \leq N-5) \\
\mathcal{O}_{e^{N-4}} & =X^{N-4}-24 q \\
\mathcal{O}_{e^{N-3}} & =X^{N-3}-128 X q \\
\mathcal{O}_{e^{N-2}} & =X^{N-2}-232 X^{2} q \\
& \\
& \\
& \\
& \\
& \\
& \\
& \\
& \\
& \\
&
\end{aligned}
$$




$$
\begin{aligned}
& H_{q, e}^{*}\left(M_{7}^{5}\right) \\
& f_{\text {rel }}(X)=X^{6}-5^{5} X^{4} q \\
& \mathcal{O}_{e^{0}}=1 \\
& \mathcal{O}_{e}=X \\
& \mathcal{O}_{e^{2}}=X^{2}-120 q \\
& \mathcal{O}_{e^{3}}=X^{3}-890 X q \\
& \mathcal{O}_{e^{4}}=X^{4}-2235 X^{2} q-49800 q^{2} \\
& \mathcal{O}_{e^{5}}=X^{5}-3005 X^{3} q-57000 X q^{2} \\
& H_{q, e}^{*}\left(M_{8}^{5}\right) \\
& f_{r e l}(X)=X^{7}-5^{5} X^{4} q \\
& \mathcal{O}_{e^{0}}=1 \\
& \mathcal{O}_{e}=X \\
& \mathcal{O}_{e^{2}}=X^{2} \\
& \mathcal{O}_{e^{3}}=X^{3}-120 q \\
& \mathcal{O}_{e^{4}}=X^{4}-890 X q \\
& \mathcal{O}_{e^{5}}=X^{5}-2235 X^{2} q \\
& \mathcal{O}_{e^{6}}=X^{6}-3005 X^{3} q-7200 q^{2} \\
& \begin{array}{rll}
H_{q, e}^{*}\left(M_{N}^{5}\right) & (N \leq 9) & \\
f_{r e l}(X) & = & X^{N-1}-5^{5} X^{4} q \\
\mathcal{O}_{e^{\alpha}} & = & X^{\alpha} \quad(0 \leq \alpha \leq N-6) \\
\mathcal{O}_{e^{N-5}} & = & X^{N-5}-120 q \\
\mathcal{O}_{e^{N-4}} & = & X^{N-4}-890 X q \\
\mathcal{O}_{e^{N-3}} & = & X^{N-3}-2235 X^{2} q \\
\mathcal{O}_{e^{N-2}} & = & X^{N-2}-3005 X^{3} q
\end{array}
\end{aligned}
$$




$$
\begin{aligned}
H_{q, e}^{*}\left(M_{8}^{6}\right) & \\
f_{r e l}(X)= & X^{7}-6^{6} X^{5} q \\
\mathcal{O}_{e^{0}}= & 1 \\
\mathcal{O}_{e}= & X \\
\mathcal{O}_{e^{2}}= & X^{2}-720 q \\
\mathcal{O}_{e^{3}}= & X^{3}-6984 X q \\
\mathcal{O}_{e^{4}}= & X^{4}-23328 X^{2} q-7076160 q^{2} \\
\mathcal{O}_{e^{5}}= & X^{5}-39672 X^{3} q-24388128 X q^{2} \\
\mathcal{O}_{e^{6}}= & X^{6}-45936 X^{4} q-9720000 X^{2} q^{2} \\
& -5094835200 q^{3}
\end{aligned}
$$

$$
\begin{aligned}
H_{q, e}^{*}\left(M_{9}^{6}\right) & \\
f_{r e l}(X) & =X^{8}-6^{6} X^{5} q \\
\mathcal{O}_{e^{\alpha}} & =X^{\alpha} \quad(1 \leq \alpha \leq 2) \\
\mathcal{O}_{e^{3}} & =X^{3}-720 q \\
\mathcal{O}_{e^{4}} & =X^{4}-6984 X q \\
\mathcal{O}_{e^{5}} & =X^{5}-23328 X^{2} q \\
\mathcal{O}_{e^{6}} & =X^{6}-39672 X^{3} q-2384640 q^{2} \\
\mathcal{O}_{e^{6}} & =X^{7}-45936 X^{4} q-2643840 X q^{2}
\end{aligned}
$$

$$
\begin{aligned}
H_{q, e}^{*}\left(M_{10}^{6}\right) & \\
f_{\text {rel }}(X) & =X^{9}-6^{6} X^{5} q \\
\mathcal{O}_{e^{\alpha}} & =X^{\alpha} \quad(1 \leq \alpha \leq 3) \\
\mathcal{O}_{e^{4}} & =X^{4}-720 q \\
\mathcal{O}_{e^{5}} & =X^{5}-6984 X q \\
\mathcal{O}_{e^{6}} & =X^{6}-23328 X^{2} q \\
\mathcal{O}_{e^{7}} & =X^{7}-39672 X^{3} q \\
\mathcal{O}_{e^{6}} & =X^{8}-45936 X^{4} q-259200 X q^{2}
\end{aligned}
$$




$$
\begin{aligned}
H_{q, e}^{*}\left(M_{9}^{7}\right) & \\
f_{r e l}(X)= & X^{8}-7^{7} X^{6} q \\
\mathcal{O}_{e^{0}}= & 1 \\
\mathcal{O}_{e}= & X \\
\mathcal{O}_{e^{2}}= & X^{2}-5040 q \\
\mathcal{O}_{e^{3}}= & X^{3}-61236 X q \\
\mathcal{O}_{e^{4}}= & X^{4}-261688 X^{2} q-1045981440 q^{2} \\
\mathcal{O}_{e^{5}}= & X^{5}-561855 X^{3} q-7364461860 X q^{2} \\
\mathcal{O}_{e^{6}}= & X^{6}-762307 X^{4} q-8660264508 X^{2} q^{2} \\
& -53577635146560 q^{3} \\
\mathcal{O}_{e^{7}}= & X^{7}-818503 X^{5} q-1785767760 X^{3} q^{2} \\
& -47590972087680 q^{3}
\end{aligned}
$$

$$
\begin{aligned}
H_{q, e}^{*}\left(M_{10}^{7}\right) & \\
f_{r e l}(X) & =X^{9}-7^{7} X^{6} q \\
\mathcal{O}_{e^{0}} & =1 \\
\mathcal{O}_{e} & =X \\
\mathcal{O}_{e^{2}} & =X^{2} \\
\mathcal{O}_{e^{3}} & =X^{3}-5040 q \\
\mathcal{O}_{e^{4}} & =X^{4}-61236 X q \\
\mathcal{O}_{e^{5}} & =X^{5}-261688 X^{2} q \\
\mathcal{O}_{e^{6}} & =X^{6}-561855 X^{3} q-579121200 q^{2} \\
\mathcal{O}_{e^{7}} & =X^{7}-762307 X^{4} q-1874923848 X q^{2} \\
\mathcal{O}_{e^{8}} & =X^{8}-818503 X^{5} q-739786320 X^{2} q^{2}
\end{aligned}
$$




$$
\begin{aligned}
H_{q, e}^{*}\left(M_{11}^{8}\right) & \\
f_{r e l}(X) & =X^{10}-8^{8} X^{7} q \\
\mathcal{O}_{e^{0}} & =1 \\
\mathcal{O}_{e}= & X \\
\mathcal{O}_{e^{2}}= & X^{2} \\
\mathcal{O}_{e^{3}}= & X^{3}-40320 q \\
\mathcal{O}_{e^{4}}= & X^{4}-594432 X q \\
\mathcal{O}_{e^{5}}= & X^{5}-3146624 X^{2} q \\
\mathcal{O}_{e^{6}}= & X^{6}-8388608 X^{3} q-134298823680 q^{2} \\
\mathcal{O}_{e^{7}}= & X^{7}-13630592 X^{4} q-875510074368 X q^{2} \\
\mathcal{O}_{e^{8}}= & X^{8}-16182784 X^{5} q-994943923200 X^{2} q^{2} \\
\mathcal{O}_{e^{9}}= & X^{9}-16736896 X^{6} q-203929850880 X^{3} q^{2} \\
& -5414928570777600 q^{3}
\end{aligned}
$$




\section{References}

[1] M.Kontsevich. Enumeration of Rational Curves via Torus Actions hep-th 9405035

[2] A.Bertram. Modular Schubert Calculus University of Utah Preprint (1994)

[3] J.Li, G.Tian. Quantum Cohomology of Homogeneous Varieties Preprint

[4] K.Intrigator. Fusion residues Modern Physics letters A6 (1991), Number 38, pp. 3543-3556.

[5] M.Jinzenji. Construction of Free Energy of Calabi-Yau Manifold embedded in $C P^{N-1}$ via Torus Actions UT-HEP-703-95

[6] M.Nagura and K.Sugiyama, "Mirror Symmetry of K3 Surface", UT-663, to appear in Int.J. of Mod. Phys.

[7] B.R. Greene, D.R. Morrison and M.R. Plesser, Mirror Manifolds in Higher Dimension, CLNS-93/1253, IASSNS-HEP-94/2, YCTP-P31-92

[8] P. Candelas and X. de la Ossa, Nucl. Phys. B355 (1991) 415.

[9] P. Candelas, X. de la Ossa, P. Green and L. Parkes, Phys. Lett. 258B (1991) 118; Nucl. Phys. B359 (1991) 21.

[10] M.Bershadsky, S.Cecotti, H.Ooguri and C.Vafa. Kodaira-Spencer Theory of Gravity and Exact Results for Quantum String Amplitudes Nucl. Phys. B405 (1993) 279

[11] V.V.Batyrev. Dual polyhedra and mirror symmetry for Calabi-Yau hypersurfaces in toric varieties Preprint, Universität-GHS-Essen,1992

[12] S.Hosono, A.Klemn, S.Theisen and S.T.Yau Mirror Symmetry, Mirror Map and Applications to Calabi-Yau Hypersurfaces HUTMP-93/0801,hepth-9308122

[13] P. Griffiths and J. Harrith, Principles of Algebraic Geometry, ( Wiley, 1978 )

[14] S. Katz "Rational curves on Calabi-Yau manifolds: verifying predictions of Mirror Symmetry" Oklahoma State University preprint OSU-M-92-3,1992

[15] M.Kontsevich , Y.Manin. Gromov-Witten Classes, Quantum Cohomology,and Enumerative Geometry hepth-9402147

[16] S.Keel. Intersection theory of moduli spaces of n-stable pointed curves of genus zero Trans. Ams, 330 (1992), 545-574.

[17] C.Itzykson. Counting rational curves on rational surfaces Saclay preprint T94/001 
[18] S.Cecotti,C.Vafa. Nuclear Physics B367 (1991) 359;

[19] B.Dubrovin. Geometry of 2D Topological Field Theory Preprint SISSA89/94/FM hepth 9407018

[20] C.Vafa. Topological Mirrors and Quantum Rings HUTP-91/A059

[21] M.Jinzenji and M.Nagura Mirror Symmetry and An Exact Calculation of $N-2$ point Correlation Function on Calabi-Yau Manifold embedded in $C P^{N-1}$ UT-680

[22] B.R.Greene, D.R.Morrison and M.R.Plesser Mirror Manifolds in Higher Dimension CLNS-93/1253,IASSNS-HEP-HEP-94/2,YCTP-P31-92

[23] E.Witten Mirror Manifolds and Topological Field Theory in Essays on Mirror Manifolds, ed. S.-T.Yau (Int. Press. Co.,Hong Kong, 1992) 120-180

[24] P.Griffiths and J.Harrith Principles of Algebraic Geometry Wiley 1978

[25] R.Dijkgraaf Intersection Theory, Integrable Hierarchies and Topological Field Theory IASSNS-HEP-91/91

[26] E.Witten On the topological phase of two dimensional gravity Nucl.Phys.B340 (1990) 281

[27] M.Kontsevich Intersection theory on the moduli space of curves and the matrix Airy function Commun.Math.Phys.147 (1992),1-23

[28] D.R.Morrison and M.R.Plesser Summing the Instantons: Quantum Cohomology and Mirror Symmetry in Toric Varieties DUKE-TH-94-78 IASSNS-HEP-94/82 hep-th/9412236

[29] T.Eguchi, S.K.Yang The Topological $C P^{1}$ Model And The Large N Matrix Integral Phys. Letters A, Vol. 9, No. 31 (1994) 2893-2902

[30] K.Hori Constraints for Topological Strings in D $\geq 1$ UT-694

[31] P.S.Aspinwall and D.R.Morrison Topological field theory and rational curves Commun.Math.Phys. 151 (1993),245-262

[32] A. Beauville Quantum cohomology of complete intersections. Preprint, 1995, alg-geom/9501008.

[33] A. Collino private communication

[34] A.B.Givental Equivariant Gromov-Witten Invariants alg-geom/9603021

[35] M.Jinzenji in preparation 